\title{
ONGOING AND CO-EVOLVING STAR FORMATION IN zCOSMOS GALAXIES HOSTING ACTIVE GALACTIC NUCLEI
}

J. D. Silverman ${ }^{1}$, F. Lamareille ${ }^{2}$, C. Maier ${ }^{1}$, S. J. Lilly ${ }^{1}$, V. Mainieri ${ }^{3}$, M. Brusa ${ }^{4}$, N. Cappelluti ${ }^{4}$, G. Hasinger ${ }^{4}$, G. Zamorani ${ }^{5}$, M. Scodeggio ${ }^{6}$, M. Bolzonella ${ }^{5}$, T. Contini ${ }^{2}$, C. M. CARollo ${ }^{1}$, K. JahnKe ${ }^{7}$, J.-P. Kneib ${ }^{8}$, O. Le Fèvre ${ }^{8}$, A. Merloni ${ }^{4,9}$, S. Bardelli ${ }^{5}$, A. Bongiorno ${ }^{4}$, H. Brunner ${ }^{4}$, K. CaPuti ${ }^{1}$, F. Civano ${ }^{10}$, A. Comastri ${ }^{5}$, G. Coppa ${ }^{5}$, O. Cucciati ${ }^{11}$, S. De la Torre ${ }^{8}$, L. De Ravel ${ }^{8}$, M. Elvis ${ }^{10}$, A. Finoguenov ${ }^{4}$, F. Fiore ${ }^{12}$, P. Franzetti ${ }^{6}$, B. Garilli ${ }^{6}$, R. Gilli $^{5}$, A. Iovino ${ }^{11}$, P. KampczyK ${ }^{1}$, C. Knobel ${ }^{1}$, K. KovaČ ${ }^{1}$, J.-F. Le Borgne ${ }^{2}$, V. Le Brun ${ }^{8}$, M. Mignoli ${ }^{5}$, R. Pello ${ }^{2}$, Y. Peng ${ }^{1}$, E. Perez Montero ${ }^{2}$, E. Ricciardellit ${ }^{13}$, M. Tanaka ${ }^{3}$, L. Tasca ${ }^{8}$, L. Tresse ${ }^{8}$, D. Vergani ${ }^{5}$, C. Vignali ${ }^{14}$, E. Zucca ${ }^{5}$,

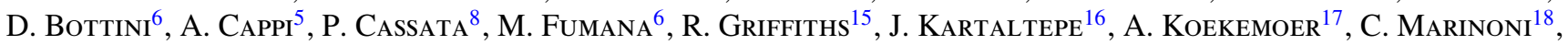
H. J. McCracken ${ }^{19}$, P. Memeo ${ }^{6}$, B. Meneux ${ }^{4,20}$, P. Oesch $^{1}$, C. Porciani ${ }^{1}$, and M. Salvato ${ }^{21}$

${ }^{1}$ Institute of Astronomy, ETH Zürich, CH-8093, Zürich, Switzerland

${ }^{2}$ Laboratoire d'Astrophysique de Toulouse-Tarbes, Université de Toulouse, CNRS, 14 avenue Edouard Belin, F-31400 Toulouse, France

${ }^{3}$ European Southern Observatory, Karl-Schwarzschild-Strasse 2, Garching, D-85748, Germany

${ }^{4}$ Max-Planck-Institut für extraterrestrische Physik, D-84571 Garching, Germany

5 INAF Osservatorio Astronomico di Bologna, via Ranzani 1, I-40127, Bologna, Italy ${ }^{6}$ INAF - IASF Milano, Milan, Italy

${ }^{7}$ Max-Planck-Institut für Astronomie, Königstuhl 17, D-69117 Heidelberg, Germany

${ }^{8}$ Laboratoire d'Astrophysique de Marseille, Marseille, France

${ }^{9}$ Excellence Cluster Universe, Boltzmannstrasse 2, D-85748, Garching beu Muenchen, Germany

${ }^{10}$ Harvard-Smithsonian Center for Astrophysics, 60 Garden Street, Cambridge, MA 02138, USA ${ }^{11}$ INAF Osservatorio Astronomico di Brera, Milan, Italy

12 INAF, Osservatorio di Roma, Monteporzio Catone (RM), Italy

13 Dipartimento di Astronomia, Universita di Padova, Padova, Italy

14 Dipartimento di Astronomia, Universitá di Bologna, via Ranzani 1, I-40127, Bologna, Italy

15 Department of Physics, Carnegie Mellon University, 5000 Forbes Avenue, Pittsburgh, PA 15213, USA

${ }^{16}$ Institute for Astronomy, University of Hawaii, 2680 Woodlawn Drive, Honolulu, HI 96822, USA

17 Space Telescope Science Institute, 3700 San Martin Drive, Baltimore, MD 21218, USA 18 Centre de Physique Theorique, Marseille, Marseille, France

${ }^{19}$ Institut d'Astrophysique de Paris, UMR 7095 CNRS, Université Pierre et Marie Curie, 98 bis Boulevard Arago, F-75014 Paris, France

${ }^{20}$ Universitats-Sternwarte, Scheinerstrasse 1, D-81679 Muenchen, Germany

${ }^{21}$ California Institute of Technology, Pasadena, CA, USA

Received 2008 July 15; accepted 2009 January 13; published 2009 April 15

\section{ABSTRACT}

We present a study of the host galaxies of active galactic nucleus (AGN) selected from the zCOSMOS survey to establish if accretion onto supermassive black holes (SMBHs) and star formation are explicitly linked up to $z \sim 1$. We identify 152 galaxies that harbor AGN, based on their X-ray emission $\left(L_{0.5-10 \mathrm{keV}}>10^{42} \mathrm{erg} \mathrm{s}{ }^{-1}\right)$ detected by XMM-Newton observations of 7543 galaxies $\left(i_{\text {acs }}<22.5\right)$. Star formation rates (SFRs), including those weighted by stellar mass, of a subsample are determined using the [O II] $\lambda 3727$ emission-line luminosity, corrected for an AGN contribution based on the observed [O III] 55007 strength or that inferred by their hard (2-10 keV) X-ray luminosity. We find that an overwhelming majority of AGN host galaxies have significant levels of star formation with a distribution spanning $\sim 1-100 M_{\odot} \mathrm{yr}^{-1}$; their average SFR is higher than that of galaxies with equivalent stellar mass $\left(M_{*}>4 \times 10^{10} M_{\odot}\right)$. The close association between AGN activity and star formation is further substantiated by an increase in the fraction of galaxies hosting AGN with the youthfulness of their stars as indicated by the rest-frame color $(U-V)$ and spectral index $D_{n}(4000)$; we demonstrate that a mass-selected sample is required to alleviate an artificial peak in the AGN fraction falling in the transition region due to the fact that many "blue cloud" galaxies have low mass-to-light ratios in luminosity-limited samples. We also find that the SFRs of AGN hosts evolve with cosmic time in a manner that closely mirrors the overall galaxy population and naturally explains the low SFRs in AGNs $(z<0.3)$ from the SDSS. We conclude that the conditions most conducive for AGN activity are a massive host galaxy and a large reservoir of gas. Furthermore, a direct correlation between mass-accretion rate onto SMBHs and SFR is shown to be weak although the average ratio $\left(\sim 10^{-2}\right)$ is constant with redshift, effectively shifting the evidence for a co-evolution scenario in a statistical manner to smaller physical scales (i.e., within the same galaxies). The order-of-magnitude increase in this ratio compared to the locally measured value of $M_{\mathrm{BH}} / M_{\text {bulge }}$ is consistent with an AGN lifetime substantially shorter than that of star formation. Our findings illustrate an intermittent scenario with underlying complexities regarding fueling over vastly different physical (and temporal) scales yet to be firmly determined.

Key words: galaxies: active - galaxies: evolution - quasars: emission lines - quasars: general - X-rays: galaxies

\section{INTRODUCTION}

The remarkable similarity between the evolution of the star formation history of galaxies and the emissivity of AGN from $z \sim 1$ to the present (e.g., Boyle \& Terlevich 1998; Franceschini et al. 1999; Merloni et al. 2004; Shankar et al. 2009; Silverman et al. 2008b) suggests a common mechanism that regulates 
their growth. Major mergers of galaxies (Mihos \& Hernquist 1996) and secular evolution (Kormendy \& Kennicutt 2004; Hopkins \& Hernquist 2006) can both potentially funnel gas to the nuclear region that can then power concurrent star formation and accretion onto supermassive black holes. Fully consistent with this scheme, young stellar populations are known to be prevalent within the bulges of nearby Seyfert galaxies (e.g., Terlevich et al. 1990; González Delgado et al. 2001; Whittle \& Nelson 2003) and luminous quasars (Jahnke et al. 2004a; Letawe et al. 2007). Enhanced levels of ongoing star formation are also evident in nearby Seyfert 2s (Gu et al. 2006) and the hosts of some mid-infrared-selected quasars (Lacy et al. 2007) although are not high enough to put them in the same class as the Ultraluminous Infrared Galaxies (Sturm et al. 2006). On the other hand, the lack of star formation based on [O II] emission in the hosts of a significant sample of PG quasars (Ho 2005) and type 1 AGN (Kim et al. 2006) from the Sloan Digital Sky Survey (SDSS) may indicate an elevated role for AGN in suppressing star formation (Granato et al. 2004; Springel et al. 2005; Croton et al. 2006; Cavaliere \& Menci 2007; Hopkins et al. 2008a). However, there do appear to be high levels of star formation in the hosts of PG quasars when considering their far-infrared emission (Schweitzer et al. 2006; Netzer et al. 2007), and type 2 quasars from the $\operatorname{SDSS}(0.3<z<0.8$; Zakamska et al. 2003; Kim et al. 2006) that may hint at an underlying evolutionary sequence (i.e., a modification to the unified model with a correlation between star formation and nuclear obscuration) or the systematic increase in the global star formation history of galaxies (e.g., Lilly et al. 1995; Hopkins \& Beacom 2006; Noeske et al. 2007; Tresse et al. 2007; Zheng et al. 2007). These seemingly discrepant results highlight the complexity in determining if a casual relationship between star formation and AGN activity exists. This is most likely due to the challenges in measuring the host galaxy properties under the glare of a bright AGN, widely varying selection methods, and inadequate control samples.

The ability to study the host galaxies of AGN selected from a large parent sample of galaxies has dramatically improved in the last few years. The SDSS has generated an unprecedented sample of galaxies to select large numbers of low redshift $(z<0.3)$ narrow-line AGN (Kauffmann et al. 2003b; Kewley et al. 2006; Stasińska et al. 2006; Heckman et al. 2006; Wild et al. 2007) to cleanly study their host properties. Powerful AGN have been clearly shown to reside in bulge-dominated galaxies with young stellar populations (Kauffmann et al. 2003b). In conjunction with Galaxy Evolution Explorer (GALEX) observations, AGN have optical-ultraviolet colors placing them in an intermediate region (Martin et al. 2007; Salim et al. 2007; Schawinski et al. 2007) between the red and blue galaxy populations thus stirring interest in their relation to transitional galaxies (Hopkins et al. 2007). Remarkably, Kauffmann et al. (2007) find a strong correlation between the mass-accretion rate onto SMBHs and the presence of young stars in the bulge that is typically accompanied by a star-forming outer disk that may be supplying the nuclear fuel. It is imperative to extend such studies to epochs closer to the peak $(z \sim 1)$ of the cosmic AGN and star formation history since AGNs may have an impact on the gas content of galaxies and their subsequent demise.

$\mathrm{X}$-ray emission is a unique probe of AGN activity due to its ability to penetrate a moderately obscuring medium $\left(N_{\mathrm{H}} \lesssim\right.$ $10^{24} \mathrm{~cm}^{-2}$ ), the lack of confusion with stellar sources, and because it allows a more direct probe of the mass-accretion rate (see Brandt \& Hasinger 2005 for a review). Selection of narrow- line AGN (i.e., obscured; type 2) by X-rays (e.g., Barger et al. 2003; Szokoly et al. 2004; Silverman et al. 2005; Brusa et al. 2007) can compensate for the deficiencies of optical selection based on emission line ratios at $z>0.3$. X-ray observations with both Chandra and XMM-Newton are being exploited to cleanly study the host galaxies of an X-ray-selected AGN (Grogin et al. 2005; Nandra et al. 2007; Pierce et al. 2007; Gabor et al. 2009; Georgakakis et al. 2008). Recently, Silverman et al. (2008a) show that the bulge-dominated hosts of the X-ray-selected AGN in the Extended Chandra Deep Field-South have rest-frame colors that are bluer with increasing redshift, possibly related to the star formation history of galaxies. In a related study, the mean star formation rate based on broad-band photometry of 58 X-ray-selected AGNs $(z \sim 0.5-1.4)$ in the 1 Msec CDF-S has been shown to be similar to IRAC-selected galaxies in an equivalent mass regime (Alonso-Herrero et al. 2007). To date, few studies using a significant sample of AGN-selected from a large parent sample of galaxies with optical spectroscopy have been undertaken at these higher redshifts $(z>0.3)$.

To do so, we utilize the rich multiwavelength observations of the COSMOS field (Scoville et al. 2007) to carry out such a study at $0.5 \lesssim z \lesssim 1.0$. The COSMOS survey is roughly a $2 \mathrm{deg}^{2}$ region of the sky selected to be accessible from all major observatories both from the ground (e.g., Subaru, VLT) and space (e.g., HST, Spitzer, XMM-Newton, Chandra). The zCOSMOS survey (Lilly et al. 2007) targets objects for optical spectroscopy with the VLT in two separate observing programs. A bright sample $\left(i_{\text {acs }}<22.5\right)$ is observed with a red grism to provide a wavelength coverage of 5500-9500 $\AA$ ideal for identifying galaxies $\left(L_{*}\right)$ up to $z \sim 1.2$. A deep program, not utilized in the present study, targets faint galaxies $(B<25)$, selected to be in the redshift range $1.5 \lesssim z \lesssim 2.5$, using a blue grism with a wavelength coverage of $3600 \AA<$ $\lambda<6700 \AA$. Here, we select a sample of galaxies based on their stellar mass with reliable spectroscopic redshifts from the zCOSMOS bright program. Those that host AGN are identified by their X-ray emission as detected by XMM-Newton (Hasinger et al. 2007; Cappelluti et al. 2007). We measure the strength of emission lines (i.e., [O II] $\lambda 3727$, [O III] $\lambda 5007$ ), using our automated pipeline ("platefit_vimos;" Lamareille et al. 2009) to determine star formation rates for the entire galaxy sample including those hosting AGN. An additional spectral indicator $\left(D_{n} 4000\right)$ enables us to discern the age of the stellar populations on longer timescales. X-ray luminosities of the AGN give us a handle on their bolometric output, less affected by obscuration, to infer mass-accretion rates and determine any trends with star formation rate and redshift. Finally, we point out that a companion paper (Silverman et al. 2009) based on the zCOSMOS $10 \mathrm{k}$ catalog expands on the current study by investigating the environmental impact on AGN activity and their host galaxy properties.

Throughout this work, we assume $H_{0}=70 \mathrm{~km} \mathrm{~s}^{-1} \mathrm{Mpc}^{-1}$, $\Omega_{\Lambda}=0.75, \Omega_{\mathrm{M}}=0.25$, and AB magnitudes.

\section{DATA AND DERIVED BROADBAND PROPERTIES}

\subsection{Parent Galaxy Sample and AGN Identification}

We use the zCOSMOS $10 \mathrm{k}$ spectroscopic "bright" catalog to construct a well defined sample of galaxies including those hosting X-ray-selected AGNs up to $z \sim 1$. Specifically, we identify 7543 galaxies with a selection magnitude $i_{\text {acs }} \leqslant 22.5$ and high-quality spectra hence reliable redshifts up to $z=1.02$. 
Table 1

Galaxy and AGN Sample

\begin{tabular}{lccccc}
\hline \hline Sample & $\begin{array}{c}\text { Redshift } \\
\text { Range }\end{array}$ & $\begin{array}{c}\text { Log Mass } \\
\left(M_{*}\right)\end{array}$ & No. of Galaxies & $\begin{array}{c}\text { No. of AGNs } \\
\left(\log L_{0.5-10 \mathrm{keV})}\right.\end{array}$ & Purpose \\
\hline$\ldots$ & $0.1-1.02$ & $\ldots$ & 7543 & $152(>42.0)$ & \\
$\ldots$ & $0.1-1.02$ & $>10.6$ & 2540 & $105(>42.0)$ & Host galaxy mass distribution \\
A & $0.1-0.50$ & $\ldots$ & 3356 & $52(42.0-43.7)$ & Mass-dependent AGN fraction \\
& & & 1820 & $73(>42.0)$ & SFRs \\
B & $0.48-1.02$ & $>10.6$ & & $47(42.0-43.7)$ & Mass-dependent SFRs \\
& & & & &
\end{tabular}

A galaxy is included in our sample if the redshift has a quality flag 2.0 or higher that amounts to a confidence of 99\% for the overall sample. The redshift success rate is not strongly dependent on galaxy color over $0.5 \lesssim z \lesssim 1.0$ given the quality of the spectra and presence of strong features (i.e., $4000 \AA$ break; $\mathrm{Ca} \mathrm{H}+\mathrm{K},[\mathrm{OII}])$. Full details on data acquisition, reduction, redshift measurements and quality assurance can be found in Lilly et al. (2007) and S. J. Lilly et al. (2009, in preparation).

We use the catalog of X-ray sources (Cappelluti et al. 2007) generated from the uniform XMM-Newton coverage ( $\sim 50 \mathrm{ks}$ depth) of the COSMOS field (Hasinger et al. 2007) to identify galaxies within our parent sample that harbor AGN. The X-ray catalogue includes 1848 point-like sources detected above a given threshold using a maximum likelihood method in either the soft $(0.5-2 \mathrm{keV})$, hard $(2-10 \mathrm{keV})$ or ultra-hard $(5-10 \mathrm{keV})$ bands down to a limiting flux of $5 \times 10^{-16}$, $2 \times 10^{-15}$, and $5 \times 10^{-15} \mathrm{erg} \mathrm{cm}^{-2} \mathrm{~s}^{-1}$ in the respective band. The adopted threshold (likelihood $>10$ ) corresponds to a probability $\sim 4.5 \times 10^{-5}$ that a source is a background fluctuation. An additional 26 faint $X M M$ sources coincident with diffuse emission (A. Finoguenov et al. 2009, in preparation) were excluded. Both the soft (0.5-2.0 keV) and hard band (2.0-10.0 keV) detections comprise our AGN sample in order to include the lowto-moderate-luminosity and/or absorbed sources. The higher sensitivity of the XMM-Newton observations in the soft band enables us to probe lower luminosity AGN $\left(\log L_{X} \sim 43\right)$ with $0.8 \lesssim z \lesssim 1.0$ not sufficiently represented in the hard-band catalog.

Optical and near-infrared counterparts to the XMM-Newton $\mathrm{X}$-ray sources are found using a maximum-likelihood method (Brusa et al. 2007). Most X-ray sources (84\%) have reliable counterparts while the remaining are uncertain due to the presence of multiple objects within the X-ray error box that have similar probabilities of being the true counterpart. We use the Chandra observations (Elvis et al. 2009) that cover the central $1 \mathrm{deg}^{2}$ to further refine our identifications. We elect to include counterparts with lower confidence given that $\sim 50 \%$ of them are likely to be correct based on the overlap between $X M M$ Newton and Chandra identifications (M. Brusa 2008, private communication). All results presented here are confirmed based on the slightly smaller catalog of highly reliable counterparts.

The zCOSMOS "Bright" program provides optical spectra for 357 of the 1093 optical counterparts to X-ray sources having $i_{\text {acs }}<22.5$. Reliable spectroscopic redshifts are available for $90 \%$ of which 164 have redshifts between $0<z<1.02$. Higher redshift AGN up to $z \sim 4$ have been identified by the zCOSMOS "Bright" program but are not the focus of this study. Since a high fraction of the zCOSMOS galaxies with associated X-ray emission have detections in the hard $2-10 \mathrm{keV}$ band (84\%), we do not expect the inclusion of soft-selected sources to induce any significant bias. A subset (54\%) of the X-ray sources has been designated as "Compulsory" targets when designing the VIMOS masks which essentially lessens their randomness and results in a $71.9 \%$ sampling rate compared to the current rate of $29.8 \%$ for galaxies. We set priority to these sources since they were not included in the spectroscopic follow-up program using Magellan (Trump et al. 2007). Since the number of AGNs is not overwhelmingly large, we chose to not select a smaller pseudo-random sample but rather incorporate correction factors applied to derived measurements where applicable.

Moderate-luminosity AGN $\left(L_{X} \sim 10^{43} \mathrm{erg} \mathrm{s}^{-1}\right)$, detected in medium-to-deep X-ray observations, have been shown to be ideal laboratories for the study of their host galaxies due to their low optical brightness which in many cases is due to their nuclear obscuration (e.g., Tozzi et al. 2006; Mainieri et al. 2007). Our AGNs predominately have luminosities below that of typical QSOs $\left(L_{X}>10^{44} \mathrm{erg} \mathrm{s}^{-1}\right)$ but are the dominant contributor to the Cosmic X-ray Background (Gilli et al. 2007). In specific cases, we further select AGN based on their Xray luminosity $\left(42.0<\log L_{0.5-10.0 \mathrm{keV}}<43.7\right)$, as done in Silverman et al. (2008a), to isolate a sample for which we can cleanly determine their host properties (i.e., stellar masses, restframe $U-V, D_{n} 4000$ ). Gabor et al. (2009) have demonstrated that the optical emission from a nearly equivalent AGN sample in COSMOS is primarily attributed to their host galaxies; AGNdominated galaxies are preferentially at $z>0.8$ due to the fact that their X-ray luminosities are typically above our cutoff $\left(L_{X} \sim 10^{43.7} \mathrm{erg} \mathrm{s}^{-1}\right)$. We conclude that the derived properties of the host galaxies of AGN in zCOSMOS are likely to be reliable. Where feasible, we include higher luminosity AGN (QSOs; 16 with $L_{0.5-10 \mathrm{keV}}>10^{44} \mathrm{erg} \mathrm{s}^{-1}$ ), since we can measure star formation rates based on [O II] $\lambda 3727$ strength even under the glare of an intrinsically, bright AGN (see Section 3 for details). For these cases, we refrain from analyses based on their host properties such as mass-weighted SFR or rest-frame color. One caveat of our luminosity-selected sample is a potential bias towards specific accretion modes such as the "Seyfert mode" (Hopkins \& Hernquist 2006) or specific evolutionary stages in their fueling such as pre or postmergers. Fortunately, we can test the later since merger-driven models (Hopkins et al. 2008a) predict vastly different properties (i.e., SFRs, morphology) of the hosts of AGNs before and after the coalescence of massive disk galaxies.

We list in Table 1 the statistics of the parent galaxy sample and those hosting X-ray-selected AGNs. The redshift and X-ray luminosity distribution of the full AGN sample (152) is shown in Figure 1 up to $z=1.02$. Symbols denote their optical spectral properties with $82 \%$ lacking broad (FWHM $>1000 \mathrm{~km} \mathrm{~s}^{-1}$ ) emission lines and most characterized as narrow emissionline galaxies. As further detailed in subsequent sections, we isolate various subsamples based on redshift, galaxy mass, and AGN luminosity to optimize our analyses. For instance, 


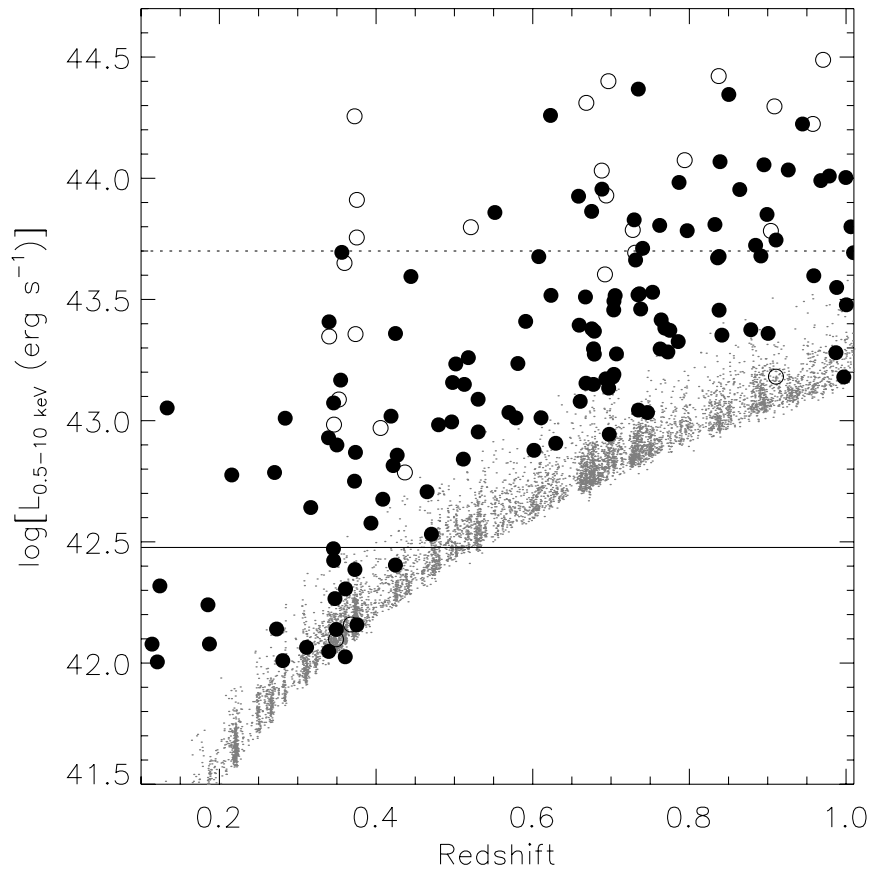

Figure 1. X-ray luminosity $(0.5-10 \mathrm{keV})$ vs. redshift for 152 AGN (large symbols). Filled circles are AGN primarily characterized by narrow-emission lines, while open symbols denote those with at least one broad optical-emission line falling within the observed spectral range. The upper limit to the broadband $\mathrm{X}$-ray luminosity at the position of each zCOSMOS galaxy, based on the X-ray sensitivity map, is shown by the small gray dots. The solid horizontal line is the minimum X-ray luminosity that we enforce to measure the fraction of galaxies hosting AGN to ensure a significant underlying galaxy population. The dotted line shows our chosen upper limit to avoid AGN contamination.

as described above, we enforce an upper limit to the X-ray luminosity ( $\left.\log L_{X}^{\max }=43.7\right)$ of the AGN when measuring a quantity with respect to its host galaxy. Also, we slightly increase our minimum X-ray luminosity $\left(\log L_{X}^{\min }=42.48\right)$ when determining the fraction of galaxies hosting AGN to ensure that a significant parent sample of zCOSMOS galaxies are capable of detecting each AGN to avoid effects based on limited statistics ${ }^{22}$.

\subsection{Stellar Mass Measurements}

Stellar masses, including rest-frame absolute magnitudes ( $\mathrm{AB}$ system; $M_{U}, M_{V}$ ), are derived from fitting stellar population synthesis models from the library of Bruzual \& Charlot (2003) to both the broadband optical (CFHT: $u, i, K_{s}$; Subaru: $B, V, g, r$, $i, z$; Capak et al. 2007) and near-infrared (Spitzer/IRAC: 3.6 $\mu$, $4.5 \mu$; Sanders et al. 2007) photometry using a chi-square minimization for each galaxy. The measurement of stellar mass $\left(\mathrm{M}_{*}\right)$ includes (1) the assumption of a Chabrier initial mass function, (2) a star formation history with both a constant rate and an additional exponentially declining component covering a range of time scales $(0.1<\tau<30 \mathrm{Gyr}),(3)$ extinction $(0<$ $A_{V}<3$ ) following Calzetti et al. (2000), and (4) solar metallicities. Further details on mass measurements can be found in

\footnotetext{
$\overline{22}$ Specifically, an overabundance of AGN at $z \sim 0.35$ falling at the flux limit of the $X M M$-Newton observations, seen in Figure 1, have an adverse effect on our determination of the fraction of galaxies hosting an AGN, due to the limited sample of zCOSMOS galaxies capable of detecting AGN with

$L_{X} \sim 10^{42} \mathrm{erg} \mathrm{s}^{-1}$, that disappears when implementing a slightly higher selection on luminosity.
}

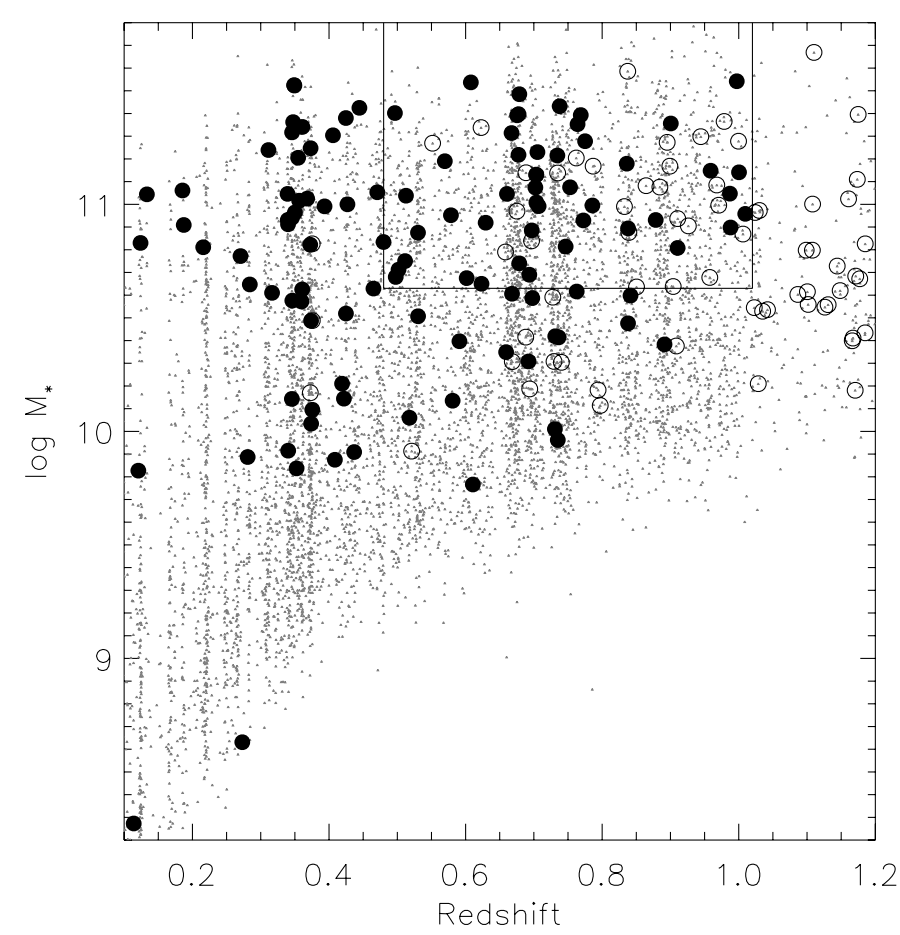

Figure 2. Stellar-mass vs. redshift for 7543 zCOSMOS galaxies (small gray circles). The box marks the mass-selected subsample of galaxies ( $\log M>$ 10.6; $0.48<z<1.02$ ) for which (specific) star formation rates are determined using $[\mathrm{O}$ II] $] \lambda 3727$. Galaxies hosting X-ray-selected AGN are further marked by a larger black circle (filled: $42.0<\log L_{0.5-10 \mathrm{keV}}<43.7$; open: $\left.\log L_{0.5-10 \mathrm{keV}}>43.7\right)$.

M. Bolzonella et al. (2009, in preparation) and B. Meneux et al. (2009, in preparation). For compatibility with the star formation rate calibration of Moustakas et al. (2006) implemented in subsequent sections, we convert all masses to an equivalent based upon a Salpeter IMF by applying a multiplicative factor of 1.7 (Pozzetti et al. 2007). We show the mass distribution for the parent galaxy population, including those harboring X-rayselected AGNs ${ }^{23}$ with $\log L_{0.5-8.0 \mathrm{keV}}>42.0$, as both a function of redshift (Figure 2) and rest-frame color ( $U-V$; Figure 3). All masses are expressed in solar units $\left(M_{\odot}\right)$ throughout this work.

We determine a minimum mass threshold that all galaxies must satisfy up to $z \sim 1.0$. The mass limit is set in order to ensure a fairly complete representation of both blue and red galaxies at all redshifts considered. In Figure 3, it is clearly evident that the mass limit of $\log M=10.6$ is essentially imposed by the red galaxy population at $z \gtrsim 0.8$ (rightmost panel). The lack of red galaxies below this limit is due to our initial selection on apparent magnitude. B. Meneux et al. (2009, in preparation) estimate based on a series of mock catalogs from the Millennium simulation that the zCOSMOS "Bright sample" is essentially complete for galaxies with $\log M \approx 10.6$ at $z=0.8$ while the completeness drops to $\sim 50 \%$ at $z=1$. In total, we have a sample of 2540 galaxies $(0.1<z<1.02)$ above this mass limit of which 105 host X-ray-selected AGNs (see Table 1 for statistics regarding subsamples employed herein.).

\footnotetext{
${ }^{23}$ It is worth mentioning that there may be a potential problem that galaxies with even moderate-luminosity AGN may have inaccurate mass estimates; a bluer continuum will essentially reduce the stellar age and hence lower the mass measurements since the derived mass-to-light ratio depends strongly on the spectrum.
} 

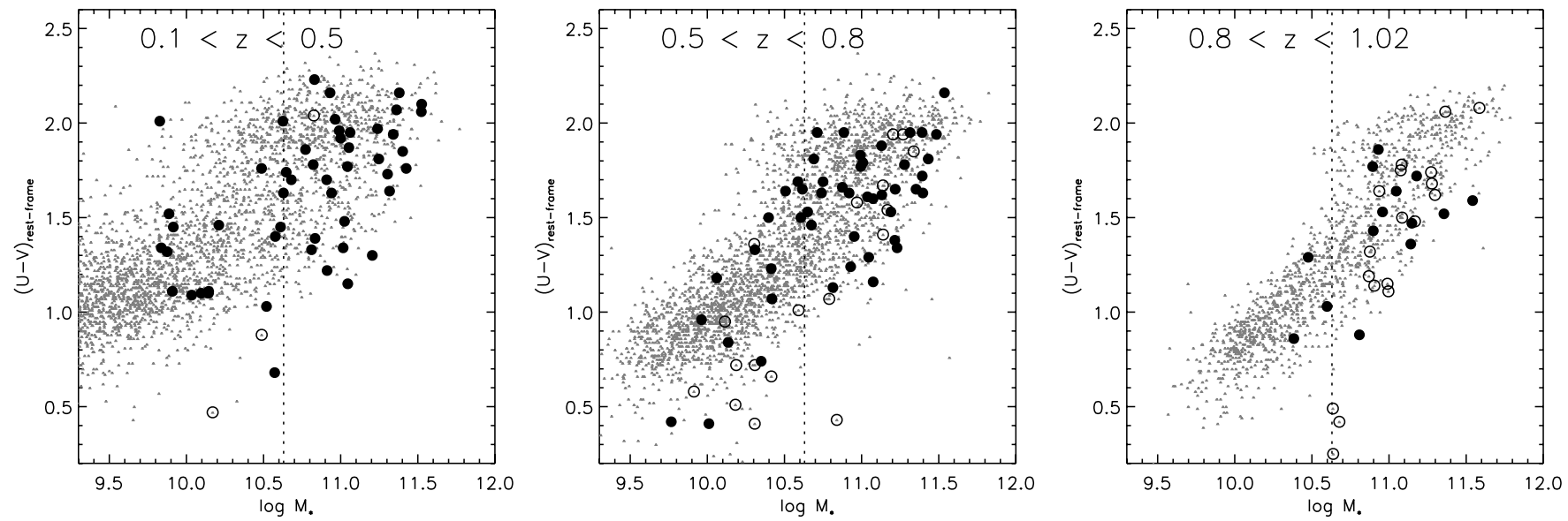

Figure 3. Rest-frame color $U-V$ vs. stellar mass split into three redshift intervals for galaxies and AGN with symbols described in Figure 2. The vertical line marks our imposed mass limit.

\section{3. [O II] AS AN SFR INDICATOR AND THE AGN CONTRIBUTION}

Our primary aim is to use the emission line [O II $] \lambda 3727$ to measure the SFRs of a well defined sample of galaxies including those hosting AGN, as commonly used for starforming galaxies at these redshifts (e.g., Kewley et al. 2004; Cooper et al. 2007; Maier et al. 2009). The availability of [O II] within the spectral window of the zCOSMOS "bright" program requires our sample of galaxies to fall within redshift range $z=0.48-1.02$. The $[\mathrm{O}$ II] line is one of a suite of spectral features (e.g., $\mathrm{H} \alpha \lambda 6563, \mathrm{H} \beta \lambda 4861$, [O III] $\lambda$ 5007) measured by an automated routine "platefit_vimos" (Lamareille et al. 2009) that simultaneously fits all lines with a Gaussian function to determine line flux and equivalent widths for the entire $\mathrm{zCOSMOS} 10 \mathrm{k}$ sample. Emission line fluxes are corrected for slit loss based on a comparison of their spectroscopic and photometric $\left(i_{A C S}\right)$ magnitudes. Line measurements with a significance less than $1.15 \sigma$ are quoted here as upper limits.

An assessment of the AGN contribution to the [O II] emission line is required even though the production of this low ionization line has been shown to be relatively weak. Croom et al. (2002) postulate, based on a comparison of the equivalent width of [O II] in the composite $2 \mathrm{dF}$ QSO spectrum to that in a composite "normal' galaxy spectrum, that [O II] is mainly produced by star-formation in the host galaxies of AGN over a wide range of absolute magnitude. The [O II] strength has been observed to be $\sim 10 \%-30 \%$ of the $[\mathrm{O}$ III $] \lambda 5007$ emission line flux (Ferland \& Osterbrock 1986; Ho 2005; Kim et al. 2006). Ho (2005) has explored the feasibility of using [O II] as an indicator of ongoing star formation for a sample of PG quasars that in many cases provided only an upper limit. Upon further investigation using emission line ratio diagnostics, Kim et al. (2006) conclude based on a sample of $\sim 3600$ type 1 AGNs $(z<0.3)$ selected from the SDSS that [O II] emission is mainly attributed to AGN photoionization and does not have a strong H II component.

The aforementioned results do not rule out the potential effectiveness of using [O II] as a SFR indicator for different samples of AGNs such those having higher luminosities, significant intrinsic obscuration or being at higher redshifts. For example, the stellar populations of "strong" type 2 AGNs from the SDSS have young ages similar to late-type galaxies (Kauffmann et al. 2003b) based on the age indicator $D_{n} 4000$. We illustrate that this is likely related to the presence of ongoing star formation. In Figure 4, we show the $[\mathrm{O} \mathrm{II}] /[\mathrm{O} \mathrm{III}]$ ratio for type 1 (panel $a$ ) and type $2\left(\right.$ panel $b^{24} ; L_{\text {[O III] }}>10^{40.58} \mathrm{erg} \mathrm{s}^{-1}$ ) AGNs from the SDSS having $z<0.3$. The data for the type 2 AGN sample is taken from the high level products available from MPA based on the SDSS DR4 release; we require an $\mathrm{S} / \mathrm{N}>3$ detection for [O II $], \mathrm{H} \beta$, [O III] and $\mathrm{H} \alpha$. First of all, it is worth highlighting that the best-fit linear relation to the type 1 AGN (panel $a$ ) illustrates a scenario where [O II] emission

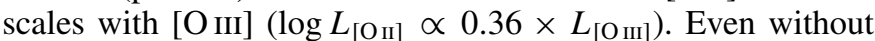
correcting for extinction, we see that there is evidence (Figure 4(b)) for elevated $[\mathrm{OII}] /[\mathrm{O} \mathrm{III}]$ for type 2 AGNs as seen by the flatter slope of the best-fit relation (slanted dashed line; $\left.\log L_{\left[\mathrm{III}_{\mathrm{II}}\right.} \propto 0.58 \times L_{\left[\mathrm{O}_{\mathrm{III}}\right]}\right)$ compared to that of the type 1 AGNs. The change in slope appears to signify that the strength of an additional component to [O II] (i.e., star formation) increases with AGN luminosity possibly related to the results of Kauffmann et al. (2007) where a decline in stellar age is seen for AGNs with higher mass-accretion rates. Finally, we see conclusively that the AGN host galaxies in zCOSMOS, while having higher [O III] luminosities, have elevated [O II]/[O III] ratios compared to type 1 AGNs (panel $a$ ), and exhibit a similar slope to the type 2 AGNs in the SDSS and slightly further enhanced [O II] emission.

When correcting for internal extinction based on the Balmer decrements, we find that the type 2 AGNs have a significantly higher distribution of $\left[\mathrm{O}_{\mathrm{II}}\right] /[\mathrm{O} \mathrm{III}]$ than the type 1s (Figure 4(c)). This further suggests that star formation is more prevalent in the type 2 AGN population compared to those with an observable broad-line region; such a hypothesis has been put forth by Kim et al. (2006) to explain the [O II] emission $(\langle[\mathrm{O} \mathrm{II}] /[\mathrm{O} \mathrm{III}]\rangle=-0.12)$ evident in the more luminous, type 2 QSOs (Zakamska et al. 2003). To further justify our use of [O II], we highlight that a correlation exists between the spectral index $D_{n} 4000$ and either the observed [O II] emission or that minus an AGN component (Figure 5) using a method fully described below thus in agreement with significant levels of ongoing star formation seen in the hosts of type 2 AGNs (Gu et al. 2006; Yan et al. 2006).

\footnotetext{
24 The luminosity selection of type 2 AGNs from the SDSS is equivalent to the definition of "strong" AGN $\left(L>10^{7} L_{\odot}\right)$ given in Kauffmann et al. (2003b). This luminosity selection, based on extinction-corrected values, guarantees that the sample is dominated by Seyfert galaxies comparable to the
} luminosities of the zCOSMOS AGN. 

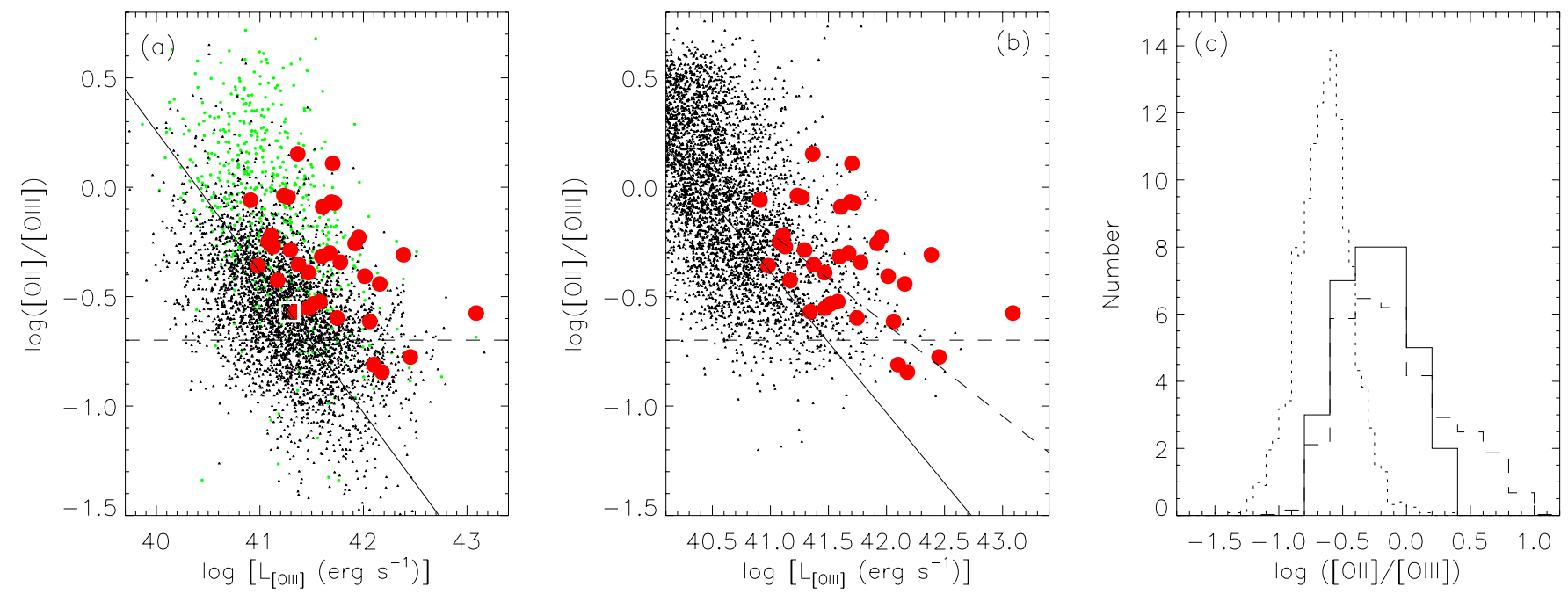

Figure 4. Emission-line properties of AGN. (a) Observed (i.e., no extinction correction) line ratio [OII]/[O III] vs. [O III] luminosity for zCOSMOS galaxies $(\log M>10.6$; small green points) and those hosting AGNs shown by larger red circles. For comparison, emission line properties of type 1 AGN from the SDSS $(z<0.3$; Kim et al. 2006) are marked by the small black dots that are further characterized by the best-fit linear relation (solid line) and mean ratio $\langle[\mathrm{O}$ II $/[\mathrm{O}$ III $]\rangle=0.27$ (open white square). The dashed horizontal line denotes our single assumption for the value of a purely AGN-dominated [O II $] /[\mathrm{O}$ III] ratio (see text for further details). (b) Same as panel (a) but with type 1 AGNs replaced by type 2 AGNs from Kauffmann et al. (2003b). The slanted lines are the best-fit linear relation to type 1 (solid) and type 2 (dashed) AGNs. (c) Histogram of the extinction-corrected [O II]/[O III] distribution for the type 1/2 AGNs (short dash/long dash; both scaled for display purposes) from the SDSS and our zCOSMOS sample (solid) all having $\log L_{[\mathrm{O} \text { III] }}>41.3$.

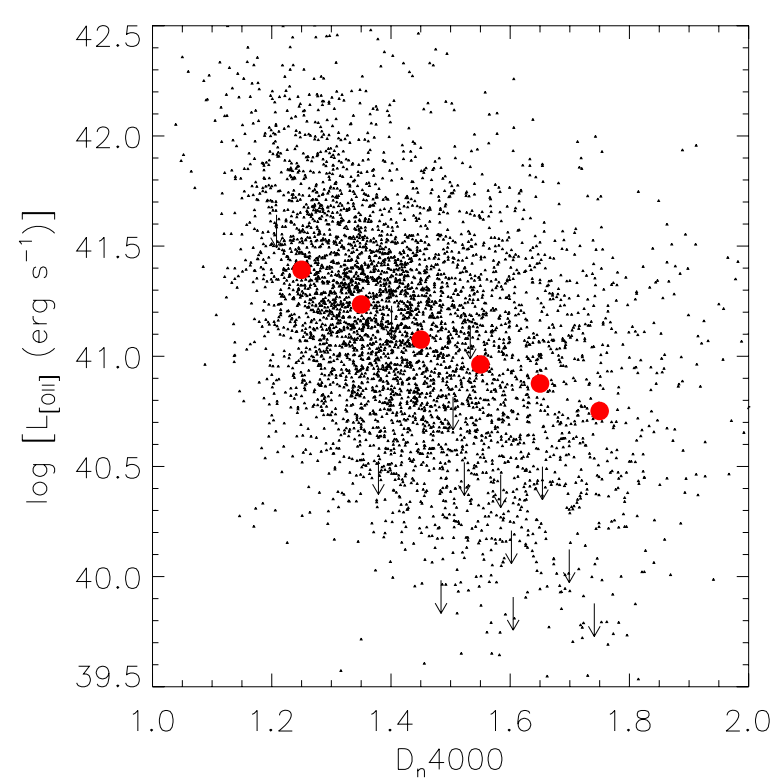

Figure 5. Relation between $D_{n} 4000$ and [O II] luminosity, corrected for an AGN component, for 5219 luminous $\left(L_{[\mathrm{O} I I I]}>10^{7} L_{\odot}\right)$, type 2 AGNs from the SDSS. Arrows mark those with upper limits. The median [O II] luminosities are shown (large red circles) in bins of $D_{n} 4000$ having a width of 0.1 .

For our purpose, we use the higher ionization line $[\mathrm{O}$ III $] \lambda 5007$ when present in our spectra, and an empirical relation between $\left[\mathrm{O}_{\mathrm{II}}\right]$ and $[\mathrm{O} \mathrm{III}]$ to statistically remove the component of the detected $[\mathrm{OII}]$ line that can be attributed to the AGN. This entails an assumption that the [O III] line is purely of AGN origin (Kauffmann et al. 2003b) that may lead us to underestimate SFRs due to a significant stellar contribution but only in very few cases since the [O III] luminosities of Seyfert galaxies in the SDSS, and also zCOSMOS AGN hosts (see Figure 4(a)), are substantially higher than that of typical H II galaxies (Kewley et al. 2006). Kim et al. (2006) find that the median value of the observed $[\mathrm{O} \mathrm{II}] /[\mathrm{O} \mathrm{III}]$ ratio is 0.27 with significant dispersion (see Figure 4(a)). We chose to use a slightly lower ratio $([\mathrm{O} \mathrm{II}] /[\mathrm{O} \mathrm{III}]=0.21)$ that is the mean value for type $1 \mathrm{AGN}$ in the SDSS sample (M. Kim 2008, private communication) with $\log L_{[\mathrm{O} \mathrm{III}]}>41.5$, a luminosity regime similar to our zCOSMOS AGN sample. This higher luminosity cut guarantees that the ratio best reflects that produced by the AGN, while effectively minimizing a contribution from $\mathrm{H}$ II regions. This results in slightly higher SFRs for our AGN sample than if we chose to use the mean of the entire type 1 SDSS sample but our final results in this study are consistent using either value. We make no attempt to use a luminosity-dependent correction, as inferred by the SDSS type 1 AGN, since after correcting the zCOSMOS sample for extinction the lowest luminosities $\left.\left(L_{[\mathrm{O}} \mathrm{III}\right]\right)$ correspond to a $[\mathrm{O} \mathrm{II}] /[\mathrm{O} \mathrm{III}]$ ratio of $\sim 0.27$, and a simple linear relation may not be evident at higher luminosities ( $\log L_{[\mathrm{O} \text { III] }}>42$ ). Also, there is no need to correct this relation for extinction given the low levels of dust attenuation $\left(A_{V} \approx 0.2 \mathrm{mag}\right.$; Kim et al. 2006) observed in these type 1 AGN. We strongly note, given the high dispersion in the $\left[\mathrm{O}_{\mathrm{II}}\right] /\left[\mathrm{O}_{\mathrm{III}}\right]$ ratio for type 1 SDSS AGN, that this is only a statistical correction applied to the population as a whole to infer the global or mean properties of the sample; the individual measurements of [O II] strength associated with star formation in a particular galaxy are expected to be inaccurate. We further note that even with this correction the more luminous AGN tend to have higher SFRs as evident by the best-fit relation for type 2 AGNs in the SDSS (Figure 4(b); $\log L_{[\mathrm{O} \text { II] }} \propto 0.37 \times L_{[\mathrm{O} \mathrm{III}]}$ ). In addition, we assess the impact of the dispersion in the $\left[\mathrm{O}_{\mathrm{II}}\right] /[\mathrm{O}$ III] ratio for type 1 SDSS AGNs by assuming a normal distribution of the line ratio $\left(\left\langle\log \left[\mathrm{O}_{\mathrm{II}}\right] /[\mathrm{O} \mathrm{III}]\right\rangle=-0.69 ; \sigma=0.25\right)$ and perform many iterations in our determination the distribution of SFR.

We consider extinction due to dust as an important factor in determining the component of the observed [O II] emission attributed to AGN photoionization given that our sample is predominantly composed of type 2 AGNs (see Figure 1). An extinction-corrected [O III] luminosity is found for each zCOSMOS AGN from the detected [O III] line emission using 

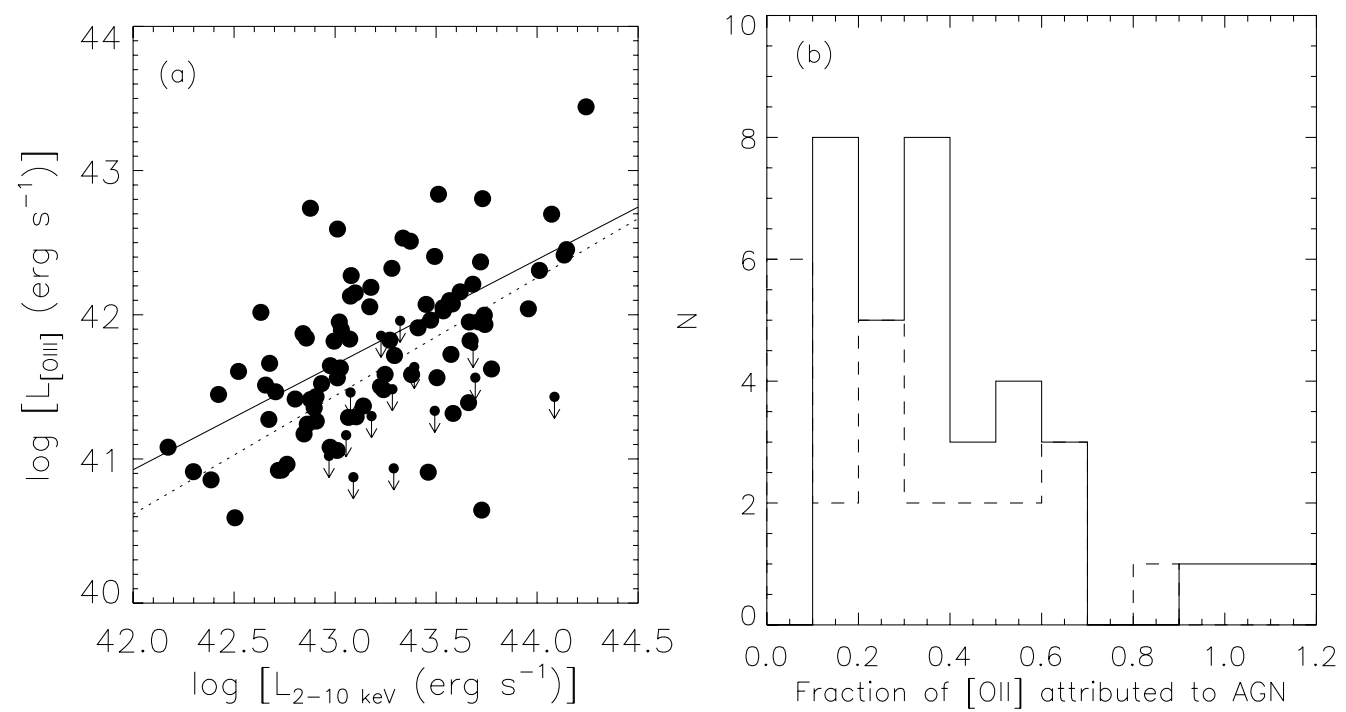

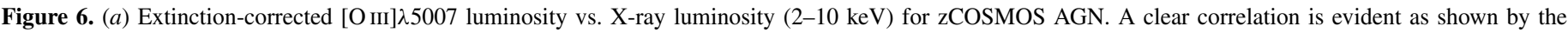

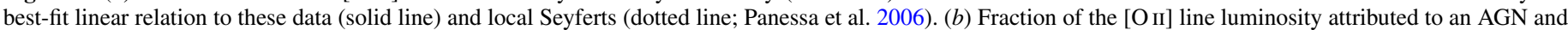

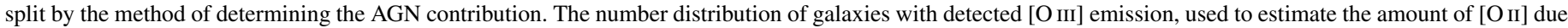

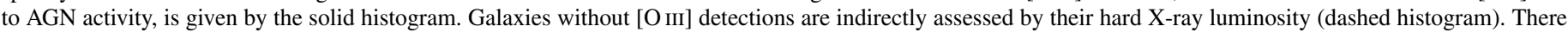
is no evidence for a systematic offset between both methods.

$A_{V}=0.8$ and the extinction law of Calzetti et al. (2000). Since most of our sample does not have $\mathrm{H} \alpha$ and $\mathrm{H} \beta$ within our observed spectral window, we chose to implement a level of extinction ${ }^{25}$ based on the mean Balmer decrement of type 2 Seyferts in the SDSS (Kewley et al. 2006). This same level of attenuation is then reapplied to the inferred AGN component to [O II], based on the empirical relation mentioned above, and subtracted from the observed (i.e., no dust correction) [O II] emission line luminosity to provide an estimate free of any AGN contribution. We are confident that this method is applicable to the zCOSMOS sample given that the [O II] luminosities of AGN hosts are all systematically higher ( 3-4 times) than that expected from gas photoionized by an AGN as indicated by the SDSS type 1 AGN (Figure 4(c)) of similar luminosities $\left.\left(\log L_{[\mathrm{O}} \mathrm{III}\right]>41.3\right)$. Also, we highlight that the enhancement of [O II] relative to [O III] for our zCOSMOS AGN is not purely induced by our extinction corrections as evident in observed relation (i.e., no extinction correction; Figures 4(a) and (b)).

For AGN at $z \gtrsim 0.8$, we need to modify our method since $[\mathrm{O} \mathrm{III}]$ is no longer within our observed spectral window. Fortunately, we can utilize the strong correlation between hard $\mathrm{X}$-ray (2-10 keV) and [O III] luminosity (Heckman et al. 2005; Panessa et al. 2006) to estimate the AGN contribution for these cases. It is important to highlight that our AGN are X-ray selected by the $0.5-10 \mathrm{keV}$ band thus limiting the inclusion of more heavily absorbed type 2 AGN such as the Comptonthick population (Fiore et al. 2009), for which this correlation is less evident if the X-ray luminosities are not corrected for $\mathrm{X}$-ray absorption (Heckman et al. 2005) as is the case here. In Figure 6(a), we plot the [O III] line luminosity, corrected for extinction as done in the previous paragraph, as a function of the rest-frame $2-10 \mathrm{keV}$ luminosity for AGN in the zCOSMOS sample with redshifts over a larger baseline $(0.2<z<1.0)$. We use these lower redshift AGN in order to provide higher statistics to measure the best-fit linear relation between these

\footnotetext{
25 This amount of optical attenuation is similar to that of X-ray-selected AGNs; we find a median $A_{V}$ of 0.98 based on the Balmer decrements of 20 AGNs in our sample with significant $\mathrm{H} \alpha$ and $\mathrm{H} \beta$ line measurements (V. Mainieri et al. 2009, in preparation)
}

two quantities. We determine the best-fit $L_{X}-L_{[\mathrm{OIII}]}$ relation (Equation (1)) for our sample by implementing a bivariate linear regression (EM) algorithm with ASURV (Survival Analysis for Astronomy package Rev. 1.2; LaValley et al. 1992) that considers limits as well as detections:

$\log L_{[\mathrm{O} \mathrm{III}]}=(0.729 \pm 0.101) \log L_{2-10 \mathrm{keV}}+(10.307 \pm 4.381)$.

This relation (Figure 6(a)), as shown by the solid line, is similar to previous studies based on a sample of low redshift AGN (e.g., Panessa et al. 2006) shown by the dotted line. We find a nearly equivalent slope although a higher normalization (i.e., larger values of $L_{[\mathrm{O} \mathrm{III}]}$ for a given $L_{2-10 \mathrm{keV}}$ ). The strong linear relation (Pearson correlation coefficient $r=0.63$ ) strengthens our assumption that most of the [O III] line luminosity is due to AGN photoionization. Any significant contribution from stars to the [O III] luminosity would increase our derived star formation rates in AGN hosts. In Figure 6(b), we demonstrate that there are no systematic offsets by introducing this indirect probe of the [O III] line luminosity; both methods essentially remove a similar fraction $(\lesssim 60 \%)$ of the [O II] luminosity attributed to AGN photoionization.

We determine SFRs using the empirical calibration of Moustakas et al. (2006). This relation considers interdependent factors (i.e., dust extinction, metallicity, and ionization) to provide a SFR tracer that is applicable to samples for which these quantities are inadequately known. To derive the following empirical relation, we fit a linear relation to the bright end $(\log L(B)>9.5)$ of the dependence of SFR on absolute $B$-band magnitude $\left(M_{B}\right.$; see Figure 19 of Moustakas et al. 2006) as implemented in Equation 2 of Maier et al. (2009):

$$
\log \mathrm{SFR}([\mathrm{O} \mathrm{II}])=\log L_{[\mathrm{O} \mathrm{II}]}-41-0.195 \times M_{B}-3.434 \text {. }
$$

The AGN component to the emission line luminosity $L_{[\mathrm{O}}$ i] is removed as described above and no correction for dust extinction is applied that is in essence an assumption that star formation is external to a dusty NLR and circumvents the potential problem that extinction of the nuclear region may differ from that of 
H II regions (see Gu et al. 2006). We do not attempt to adjust (i.e., lower) the upper limits on nondetections by considering the contribution of an AGN. It is worth noting that an additional caveat of our method is that there is an underlying assumption that the metallicity and extinction of galaxies with or without AGN are similar.

\section{PHYSICAL PROPERTIES OF AGN HOST GALAXIES}

\subsection{Stellar Masses}

We measure the fraction of galaxies that host X-ray selected AGNs as a function of their stellar mass to determine the characteristic mass above which AGN activity is most prevalent. First of all, it is apparent in Figure 2 that galaxies hosting Xray-selected AGNs are preferentially massive $(\log M \gtrsim 10.5)$ as evident over the full redshift range and in agreement with most studies to date (e.g., Kauffmann et al. 2003b; Bundy et al. 2008). For this exercise, we consider the redshift interval $0.1<z<0.5$ for which we have a high degree of completeness for galaxies over a wide mass baseline $(\log M \gtrsim 9.5)$. We follow the technique discussed in Section 3.1 of Lehmer et al. (2007) to determine the AGN fraction of our parent population of galaxies that accounts for the spatially varying sensitivity limits of the XMM observations of the COSMOS field (see Figure 17 of Cappelluti et al. 2007). The necessity of this approach is demonstrated in Figure 1, which shows the limiting $\mathrm{X}$-ray luminosity as a function of redshift for the entire galaxy sample. Even though the sensitivity of the $X M M$ coverage is remarkably uniform, some dispersion is present as shown by the relatively narrow distribution of the X-ray upper limits at each redshift. To properly account for the luminosity-redshift relation, we determine the contribution of each AGN separately to the total fraction. The AGN fraction ( $f$; see equation 3 below) is determined by summing over the full sample of AGN $(N)$ with $N_{\text {gal,i }}$ representing the number of galaxies in which we could have detected an AGN with X-ray luminosity $L_{\mathrm{X}}^{i}$. The different sampling rates, based solely on slit placement, of the galaxies $\left(S_{\text {gal }}\right)$ and AGN (i.e., X-ray sources ${ }^{26}, S_{x}$ ) are taken into account in Equation (3). We estimate the associated $1 \sigma$ error (Equation (4)) using binomial statistics where $N_{a g n}^{\text {eff }}$ is the number of AGN that would be detected if all galaxies have the same limiting X-ray sensitivity and the sample of AGN was randomly selected. Here, we only consider AGN with $42.48<\log L_{0.5-10 \mathrm{kev}}<43.7$. As previously mentioned, the lower limit ensures that we have a statistically significant sample of parent galaxies $(\gtrsim 700)$ that could host each AGN while the upper limit restricts the sample to low-to-moderate luminosities thus securing the accuracy of their host-galaxy masses. We refer the reader to Silverman et al. (2008a) for further details and results employing this method.

$$
\begin{gathered}
f=\sum_{i=1}^{N} \frac{1 / S_{x}}{N_{\text {gal }, \mathrm{i}} / S_{\text {gal }}} \\
\sigma^{2}=N_{a g n}^{\mathrm{eff}} \times\left(N_{\text {gal }}-N_{\text {agn }}^{\mathrm{eff}}\right) / N_{\text {gal }}^{3}
\end{gathered}
$$

In Figure 7, we plot the fraction of galaxies that host AGN and the number distribution (Table 1; Sample A) of both galaxies and those with AGN over a slightly wider luminosity

\footnotetext{
${ }^{26}$ In many cases, X-ray sources have redshifts based on a random placement of a slit for which the sampling reflects the overall galaxy population $\left(S_{x}=S_{\mathrm{gal}}\right)$.
}

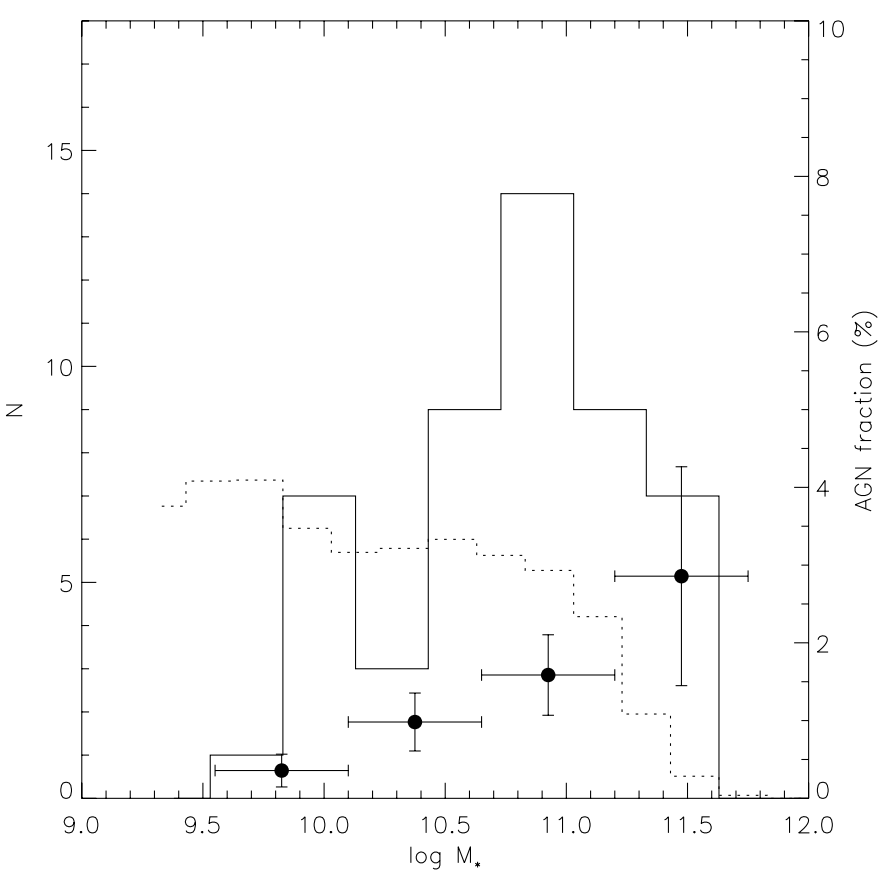

Figure 7. Stellar-mass distribution of zCOSMOS galaxies hosting AGN in the redshift range $0.1<z<0.5$. Fifty-two galaxies $\left(\log M_{*}>9.5\right)$ with an X-ray-selected AGN $\left(42<\log L_{0.5-10 \mathrm{keV}}<43.7\right)$ are shown by the solid histogram. The observed parent distribution of 3356 galaxies (dotted histogram) is renormalized to match the AGN distribution. The data points represent the AGN fraction $\left(42.48<\log L_{0.5-10 \mathrm{keV}}<43.7\right.$; scale on the right-hand vertical axis) in fixed mass intervals (shown by the horizontal bars centered on each data point) with $1 \sigma$ error bars.

interval $\left(42.0<\log L_{0.5-10 \mathrm{keV}}<43.7\right)$. The absolute fraction is low $(\sim 1-4 \%)$ due to our restrictions on X-ray luminosity. Kauffmann et al. (2003b) measure a fraction $\sim 5$ times higher although they include in their sample AGN $\sim 80$ times fainter. The difference in these fractions is mainly due to the steep faintend slope of the luminosity function at low redshifts (Shinozaki et al. 2006). Here, we are mainly interested in the relative change of the AGN fraction with galaxy mass. Clearly, we see that the fraction of galaxies that host AGN monotonically increases with stellar mass similar to results based on obscured (Kauffmann et al. 2003b) and radio-loud (Best et al. 2005) AGN in the SDSS.

\subsection{Star Formation Rates}

In Figure 8, we show the SFRs for 1820 zCOSMOS galaxies (Table 1: Sample $\mathrm{B}^{27}$ ) including 43 of them that host moderate-luminosity AGN $\left(42.0<\log L_{0.5-10.0 \mathrm{keV}}<43.7\right)$. The results are presented before (Figures $8(\mathrm{a})$ and (b)) and after (Figures 8(c) and (d)) the correction for the AGN contribution to the [O II] luminosity based on a single-value $(0.21)$ of the observed, median $[\mathrm{O} \mathrm{II}] /[\mathrm{O} \mathrm{III}]$ ratio of type 1 AGNs in the SDSS. First of all, we see that $88 \%$ of AGNs have detectable [O II] emission $^{28}$ (Figure 8(a)). It is apparent by comparing the SFR distribution for AGN hosts in Figures 8(b) and (d) that a significant shift in the distribution occurs when removing the AGN

\footnotetext{
27 The sample is slightly smaller than that given in Table 1; four AGNs have [O II] falling outside the observed spectral window that can vary slightly from slit-to-slit.

28 The fraction of AGN with detected [O II] emission is $68 \%(\mathrm{~S} / \mathrm{N}>2)$ and $61 \%(\mathrm{~S} / \mathrm{N}>3)$ depending on the given line significance. We have confirmed our results based on the lower $\mathrm{S} / \mathrm{N}$ ratio (1.15) with those based on these smaller, high-confidence samples of AGN.
} 

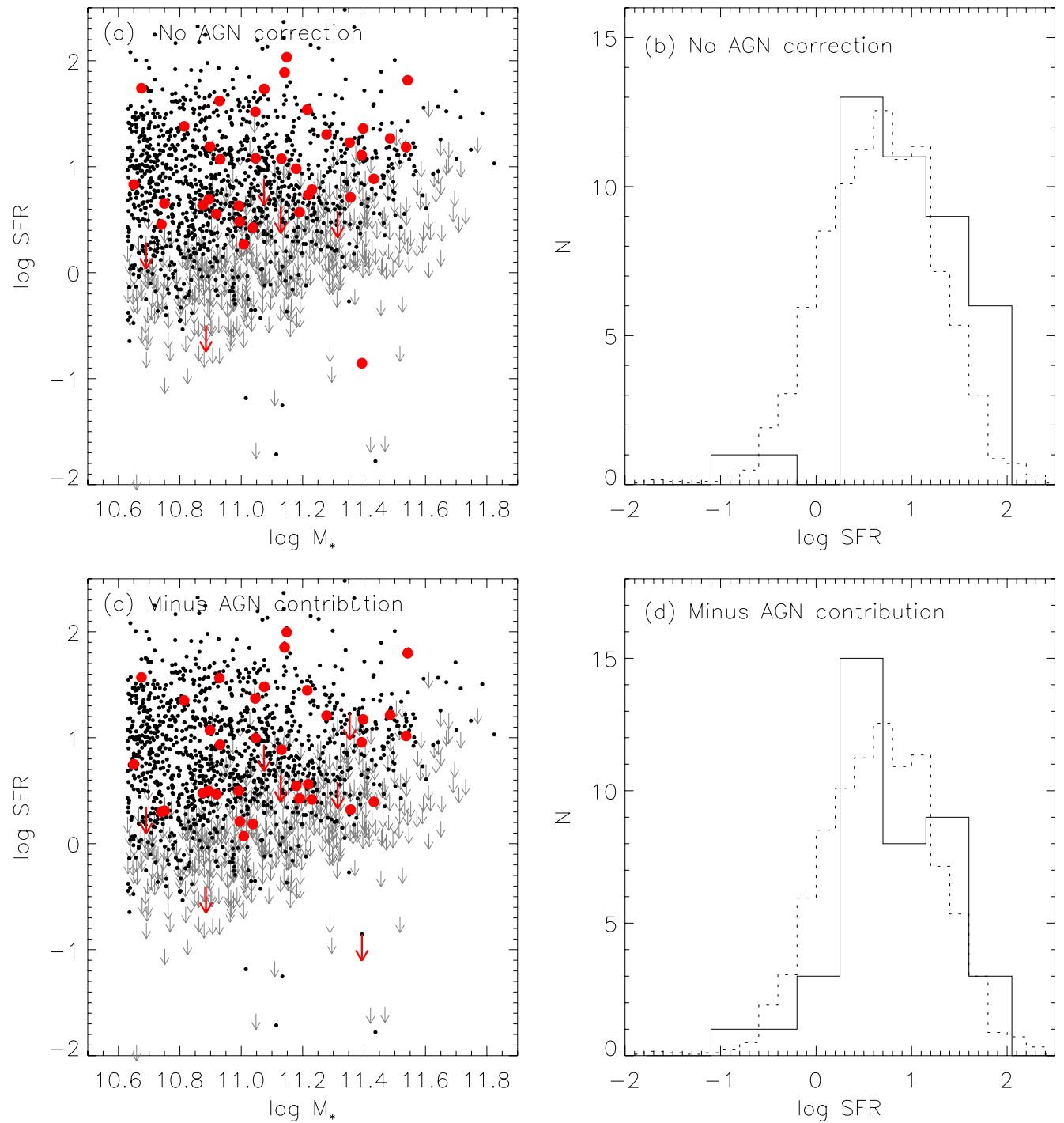

Figure 8. Star formation rates $\left(M_{\odot} \mathrm{yr}^{-1}\right)$ vs. stellar mass for galaxies $\left(0.48<z<1.02 ; \log M_{*}>10.6\right)$ before $(a)$ and after $(c)$ the removal of the AGN contribution. Measurements are shown by either a small black (emission-line galaxy) or large red (AGN; $\left.42.0<\log L_{0.5-10 \mathrm{keV}}<43.7\right)$ circle. Upper limits are shown as an arrow with their color descriptive of the type as given above. $(b, d)$ For ease of comparison, the distributions, including those with upper limits, are shown by either a solid (AGN hosts) or dashed (all galaxies) histogram with the full galaxy sample scaled down to match the AGNs.

contribution to the [O II] emission line. Even so, we find that the SFRs of AGN host galaxies are almost exclusively between 1-100 $M_{\odot} \mathrm{yr}^{-1}$ (Figures 8(c) and (d)), a range consistent with analytic models of AGN hosts with star-forming disks (Ballantyne 2008) and supportive of constraints on the cosmic IR background (Ballantyne \& Papovich 2007).

To quantitatively test whether the SFR distribution of AGN hosts differs from that of the underlying galaxy population, we have determined the SFR distribution, including those with upper limits, while also considering the dispersion in the AGN contribution to the observed [O II] emission-line luminosity. We assume a log normal distribution of the $\left[\mathrm{O}_{\mathrm{II}}\right] /[\mathrm{O} \mathrm{III}]$ ratio with parameters given in Section 3. Based on 1000 iterations, we determine the mean SFR distribution and perform statistical tests on each individual sample compared to the parent galaxy population. The results of this exercise are shown in Figure 9 for two AGN samples of differing luminosities. First, we consider AGN, as done above, that fall within the luminosity interval $42<\log L_{X}<43.7$ for which we have high confidence in our well matched, mass-selected parent sample of galaxies.
Based on this limited sample of AGNs, we cannot significantly discriminate between the two distributions (Figure 9(a)) given the results of the individual K-S tests (Figure 9(b)). Although, we do find that the median SFRs shown in Figure 9(c) are for the most part higher than that of the full galaxy sample $\left(4.7 M_{\odot}\right.$ $\mathrm{yr}^{-1}$ ) in each iteration.

We are able to use a less restrictive sample that includes higher luminosity AGN $\left(\log L_{X}>43.7\right)$ thus improving our statistics although at the expense of maintaining reliable stellar masses across our sample. In Figures 9(d)-(f), we present the equivalent analysis for AGNs with $\log L_{X}>42$. Based on this larger sample, we see that the SFR distribution of AGN hosts is shifted to higher values than the overall population (Figure 9(d)). The $\mathrm{K}-\mathrm{S}$ tests on the individual distributions now reject the null hypothesis (i.e., distributions are equivalent) at the $\sim 99 \%$ level $(>2.5 \sigma)$ in essentially all iterations (Figure 9(e)) and $65 \%$ of the iterations provide probabilities greater than that of the single comparison (0.994) without including any dispersion in the $\left[\mathrm{O}_{\mathrm{II}}\right] /[\mathrm{O} \mathrm{III}]$ relation. Moreover, the median SFRs are all systematically higher $\left(\mathrm{SFR} \sim 9.5 \mathrm{M}_{\odot} \mathrm{yr}^{-1}\right.$ ) than that of 

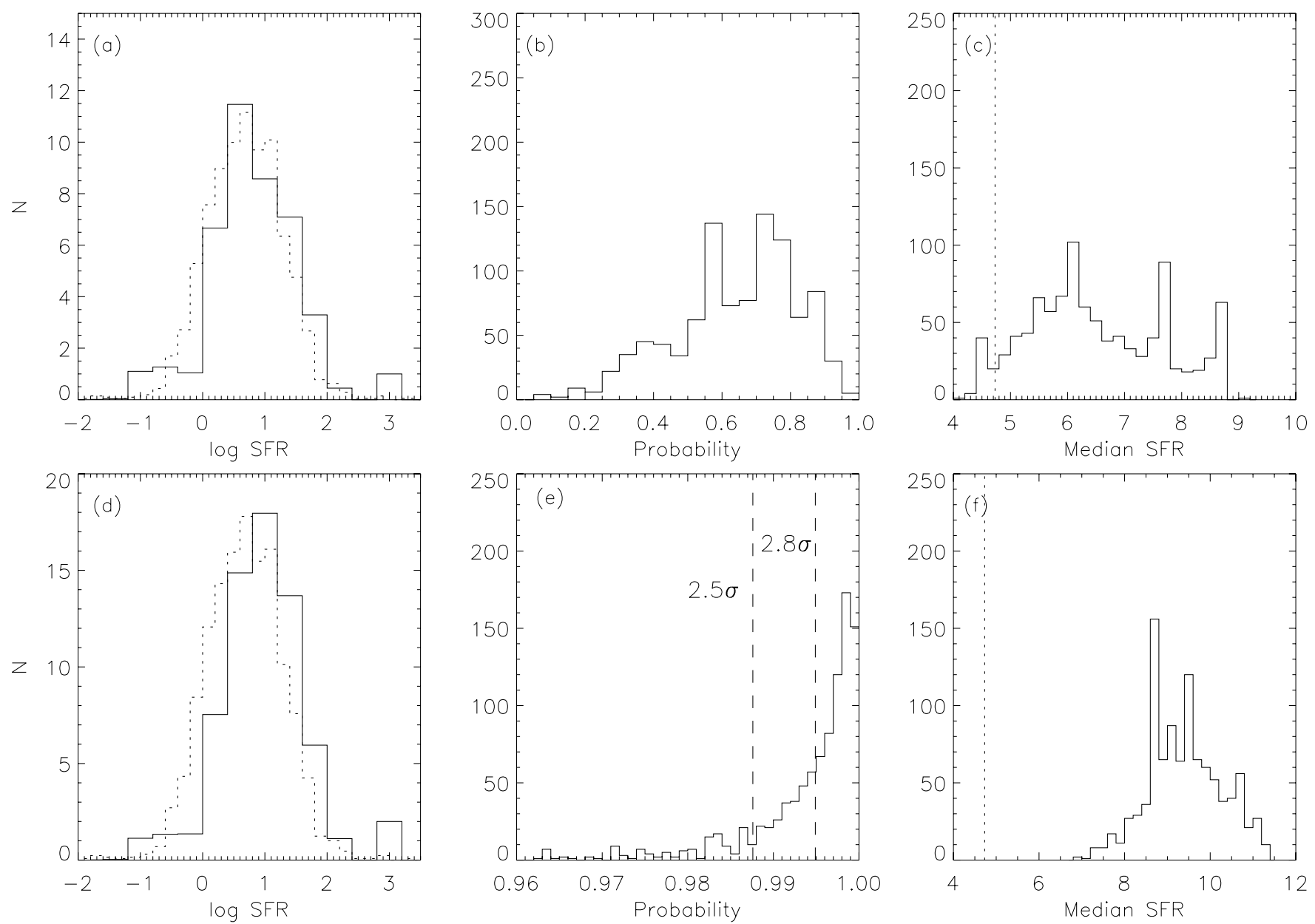

Figure 9. Star formation rates of AGN hosts with dispersion in the applied correction for the AGN contribution. The two rows are given for AGN spanning a different luminosity range: $(a)-(c) 42<\log L_{X}<43.7,(d)-(f) \log L_{X}>42$. The left panels $(a)$ and $(d)$, equivalent to those in Figure 8 , show the mean distribution of SFR, including upper limits, based on 1000 iterations. The middle panels $(b)$ and $(e)$ give the probability distribution based on KS tests that the null hypothesis (i.e., two samples are equivalent) can be rejected. The right panels $(c)$ and $(d)$ display the distribution of the median SFR for each iteration with the median SFR $\left(4.73 M_{\odot} \mathrm{yr}^{-1}\right)$ of all galaxies depicted by the vertical dashed line.

the full galaxy sample. The statistical improvement appears to not only be due to the moderate gain in sample size but have a contribution from the mild increase in SFR associated with more luminous AGN (see Figure 13(a)) or an inadequate assessment of the AGN contribution to the [O II] emission line. We further point out that the SFR distribution of AGN hosts while being elevated in comparison to the underlying massive galaxy population is essentially equivalent to those forming stars (i.e., emission-line galaxies). Therefore, we conclude that a plentiful gas reservoir is a necessary ingredient for the fueling of AGNs as indicated by the presence of significant star formation with rates reaching up to $\sim 100 M_{\odot} \mathrm{yr}^{-1}$.

We investigate how star formation in galaxies hosting AGN is evolving compared to that of the parent galaxy population. In Figure 10, we show SFR as a function of redshift for galaxies with $\log M>10.6$ and those hosting AGN (Table 1; Sample B). A systematic shift of the distribution of zCOSMOS galaxies towards higher SFRs with increasing redshift is clearly evident with those hosting AGN exhibiting a similar behavior. Therefore, we find that the SFRs of AGN hosts are dictated by that of the underlying galaxy population thus solidifying similar evidence based on the color evolution of AGN hosts in the ECDF-S (Silverman et al. 2008a). As a consequence, ongoing star formation is most likely responsible for the significant population of AGN hosts over the redshift range $0.5 \lesssim z \lesssim 1.4$ having blue rest-frame colors (Sanchez et al. 2004; Böhm et al. 2007; Nandra et al. 2007) with evidence for such a trend remaining in place for quasar hosts at higher redshifts $1.8<z<2.8$ (Jahnke et al. 2004b).

It is illuminating to compare the SFRs from zCOSMOS to AGN at lower redshifts. For consistency with zCOSMOS, we measure SFRs of the type 2 AGNs from the SDSS $(z<0.3$; Kauffmann et al. 2003b) using the [O II] 33727 and [O III] $\lambda 5007$ emission line fluxes from Brinchmann et al. (2004) to determine an AGN-corrected [O II] luminosity. We further select only those galaxies having stellar masses above our zCOSMOS limit $\left(\log M_{*}>10.6\right)$ based on the conversion factor (Kroupa to Salpeter IMF) given in Brinchmann et al. (2004). Here, we use the calibration of Kewley et al. (2004) to derive SFRs ${ }^{29}$ in order to have consistency with the SFRs measured for the type 1 SDSS sample (Kim et al. 2006). Initially, we demonstrate that the ongoing SFRs shown in Figure 10 are in agreement with the findings of Kauffmann et al. (2003b) based on $D_{n}(4000)$ : weak AGNs $\left(L_{[\mathrm{O} \text { III] }}<10^{7} L_{\odot}\right.$; blue points) have low SFRs $\left(\sim 0.2 M_{\odot} \mathrm{yr}^{-1}\right)$ while strong AGNs $\left(L_{[\mathrm{O} \text { III }]}>10^{7} L_{\odot}\right.$; small red points) are more actively forming stars $\left(\sim 1 M_{\odot} \mathrm{yr}^{-1}\right)$. For ease of visualization, we show the best-fit relation $\log$ SFR $\propto$ 29 We have confirmed that the Moustakas et al. (2006) relation gives the same
results. 


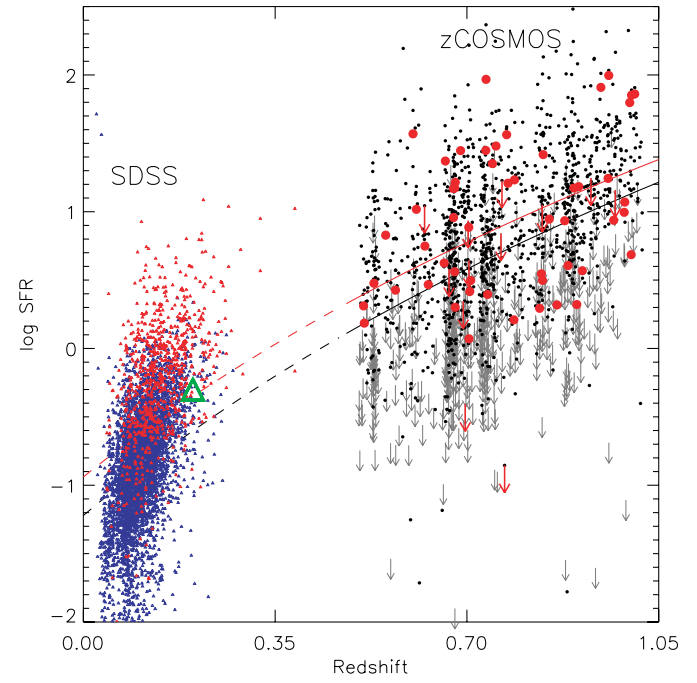

Figure 10. Cosmic evolution of star formation. At $z>0.48$, we show the SFR $-z$ distribution for all zCOSMOS galaxies with $\log M>10.6$ (small black circles and gray arrows) and those hosting AGN with $\log L_{X}>42$ (73; large red circles and arrows). The best-fit linear relation for zCOSMOS galaxies, including those with upper limits, is shown for both populations (black: galaxies; red: AGN hosts) with an extrapolation to lower redshifts (dashed lines). For comparison, we plot SFRs of AGN hosts from the SDSS with an equivalent selection on stellar mass; obscured AGNs (type 2) from the sample of Kauffmann et al. (2003b) are shown with strong AGNs $\left(\log L_{\mathrm{OIII}}>40.5\right)$ in red and those of lower luminosity in blue. A large green triangle marks the mean value of the SFR for the SDSS type 1 AGNs (Kim et al. 2006).

$\log (1+z)$ for only zCOSMOS galaxies (upper limits are included; black curve) and those hosting AGN (red curve). We note that an accurate determination of the evolution rates requires a careful assessment of all possible selection effects that is beyond the scope of the present work. Even so, we find that the SDSS AGN may be the low redshift analogs of the AGNs in the zCOSMOS survey given their close proximity to an extrapolation of the evolution of zCOSMOS galaxies. In addition, the low-to-moderate levels of star formation $(\approx 0.5$ $3 M_{\odot} \mathrm{yr}^{-1}$ ) in the type 1 SDSS AGN (shown roughly by the green triangle in Figure 10; Kim et al. 2006) are in agreement with the type 2 AGNs from the SDSS and the aforementioned evolutionary scenario of star formation in general. In light of these results from the SDSS that effectively extend our redshift baseline, we reiterate our conclusion that the SFRs of AGN host galaxies are reflective of the overall star formation history of galaxies and provide no indication of the suppression or truncation due to a mechanism related to the AGN itself.

\subsection{Stellar Ages}

To complement our study of the ongoing SFRs of AGN hosts, we use the spectral index $D_{n}$ (4000) (Balogh et al. 1999), determined by our "platefit_vimos" routine for each galaxy in the zCOSMOS sample to infer the age of the overall stellar population on longer timescales $(>0.1 \mathrm{Gyr})$. This index is the ratio of the average flux density $F_{\lambda}$ in the continuum bands 3850-3950 A and 4000-4100 $\AA$. This is essentially a measure of the strength of the $4000 \AA$ break with galaxies having experienced a recent episode of star formation exhibiting a smaller index due to the presence of young stars. In Figure 11, we show the values of $D_{n}(4000)$ for galaxies with respect to their specific SFR. We implement two mass limits $(\log M>10.6$ : Figures 11(a) and (b); $\log M>11.1$ : Figures 11(c) and (d)) in order to check for consistency when the underlying $D_{n}(4000)$ distribution of galaxies is significantly different: the higher mass cut results in a distribution dominated by more evolved galaxies as evident by its peak at $D_{n}(4000) \sim 1$.8. Initially, it is apparent (Figure 11(a)) that there is a good correspondence between $D_{n}(4000)$ and specific SFR for all galaxies including those with AGNs. We then use the relation given in Kauffmann et al. (2003a) that assumes an instantaneous burst model of solarmetallicity to infer stellar age from the value of $D_{N}(4000)$ as given by the scale bar in Figure 11(a); this relation provides us with a rough assessment of the actual ages since many of the zCOSMOS galaxies including those with AGN may have had a more sedative existence in their recent past. As a result, we find that most galaxies with AGN contain a young stellar component since $70 \%$ have $D_{n}(4000)<1.6$ (age $\lesssim 2$ Gyr; Figures 11 (a) and (c)). Furthermore, we measure the fraction of galaxies hosting an AGN as a function of $D_{n}(4000)$ and demonstrate (Figures 11(b) and (d)) that a galaxy is more likely to have an accreting SMBH if there is a sufficient supply of gas, fully consistent with the mass measurements of $\mathrm{H}_{\mathrm{I}}$ (Ho et al. 2008) and CO (Scoville et al. 2003) in AGN hosts, given the higher rate of occurrence in galaxies with younger ages. These results effectively extend such studies based on $D_{n}(4000)$ at low redshift (Kauffmann et al. 2003b) up to $z \sim 1$.

There is a notable discrepancy between our claim for a higher level of AGN activity for star-forming galaxies and the location of AGN hosts on the color-magnitude diagram. Both X-ray-selected (Nandra et al. 2007; Silverman et al. 2008a) and optically selected AGNs (Martin et al. 2007) exhibit a low AGN fraction along the red sequence, enhanced activity in the intermediate region (i.e., "green valley") between the blue and red galaxy populations, and a drop-off towards very blue galaxies. On the contrary, the mass-selected AGN sample of Kauffmann et al. (2003b) clearly have stellar properties very similar to late-type galaxies (see their Figure 14) and, thus a low fraction of AGN within the "blue cloud" reported by the aforementioned studies is surprising. Interestingly, Lehmer et al. (2008) find that the fraction of the stacked X-ray signal of latetype galaxies attributed to AGN emission continuously rises with SFR.

To investigate this further, we measure the fraction of galaxies hosting AGN as a function of their rest-frame optical color $(U-V)$ for both a luminosity and mass-selected sample (Figures 12(a) and (b)). Since we are not constrained by the use of [O II] for this exercise, we have extended the redshift baseline $0.1<z<1.02$ to improve the statistics. In Figure 12(a), we find that the fraction of galaxies with X-ray-selected AGNs is strongly peaked in the "green valley" for the luminosityselected sample thus agreeing with the results of Silverman et al. (2008a). In contrast, the mass-selected sample, behaves similarly for colors $U-V>1.4$, but does not have a decline towards bluer colors $U-V<1.4$. This is easily understood since blue galaxies are known to have lower mass-to-light ratios and the AGN fraction rises substantially with host galaxy mass as demonstrated in Section 4.1. We conclude that blue, star-forming galaxies do have enhanced levels of AGN activity and there is no evidence for a significant delay in the emergence of nuclear activity with respect to the onset of star formation. This result is consistent with the recent findings of Silverman et al. (2008a), based the morphology of the host galaxy, that blue $(U-V<0.7)$, bulge-dominated $\left(n_{\text {sersic }}>2.5\right)$ galaxies have the highest incidence $(21.3 \%)$ of AGN activity, and the blue rest-frame colors of low redshift $(z<0.2)$ quasars irrespective of their morphology (Jahnke et al. 2004a). However, there 

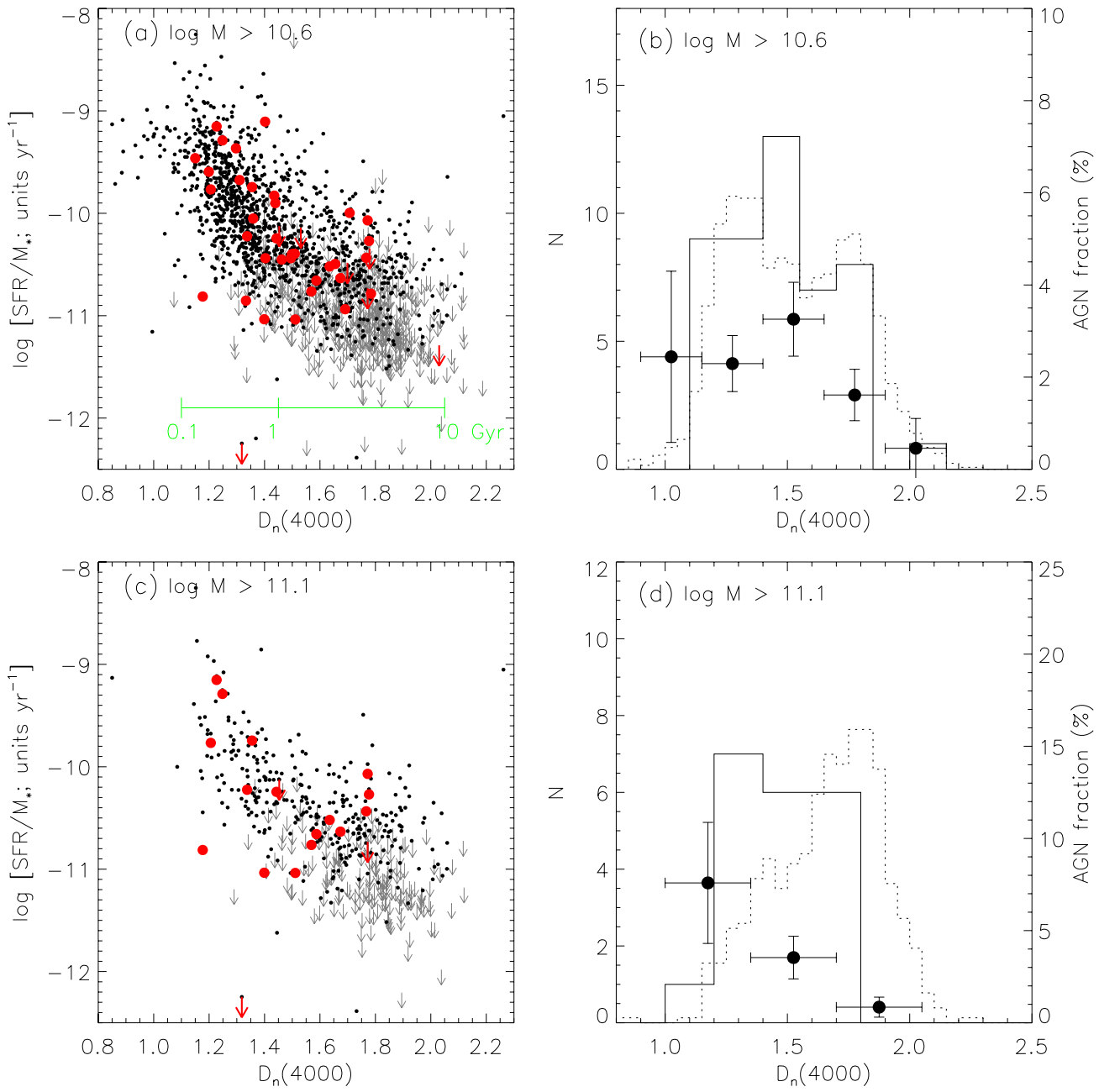

Figure 11. (a) Specific SFR vs. the stellar-age indicator $\mathrm{D}_{n}(4000)$. Symbols are equivalent to those in Figure $8(a)$. An approximate age scale is given based on Figure 2 of Kauffmann et al. (2003a). (b) Number distribution and the fraction of galaxies hosting an AGN as a function of $D_{n}(4000)$. ( $c, d$ ) The equivalent plots are shown for a higher mass cut as given. AGN activity is primarily associated with young stellar populations over a broad range in host galaxy mass.
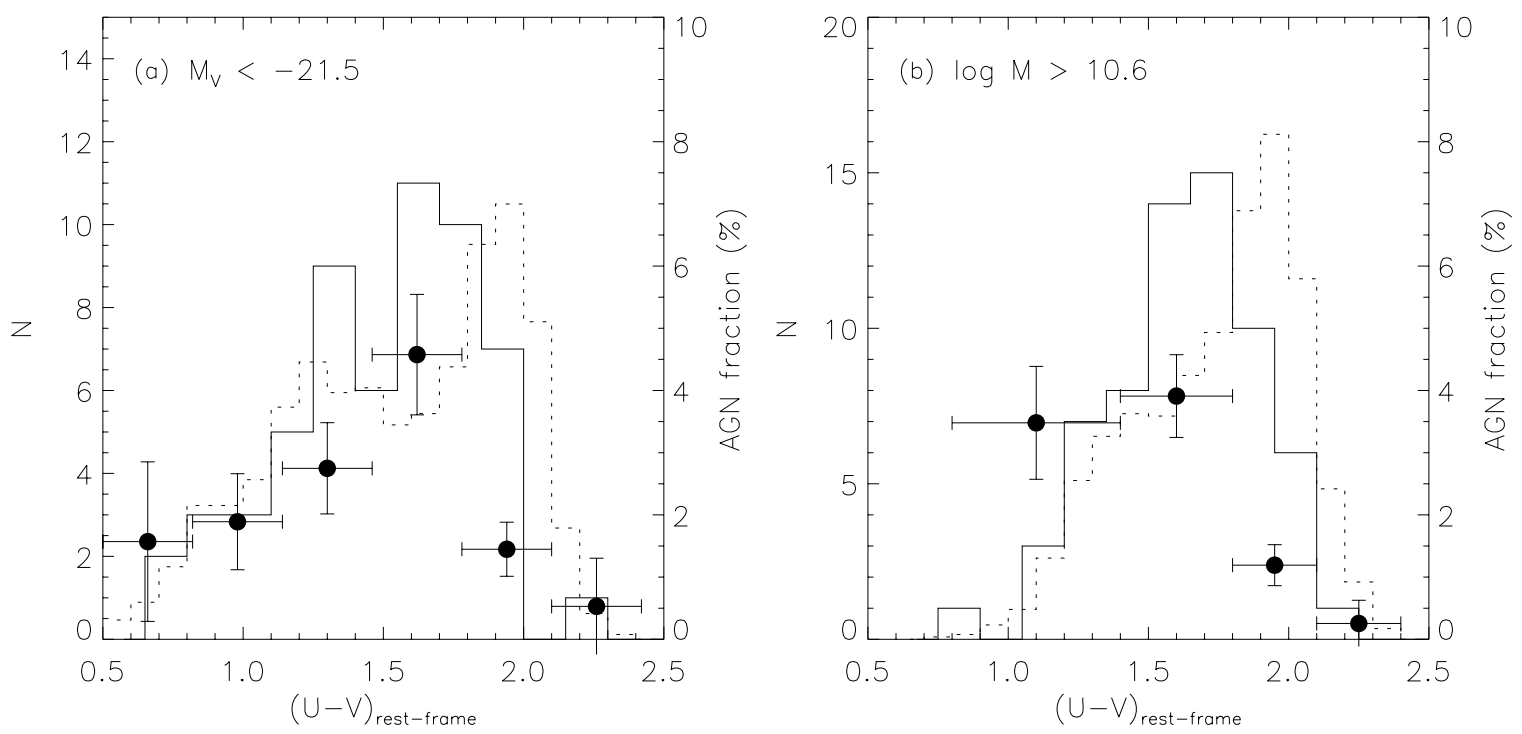

Figure 12. Rest-frame color distribution $U-V$ of galaxies $(0.1<z<1.02)$ and the fraction hosting AGN. We show the distribution of AGN hosts (solid histogram) with 58 selected by optical luminosity $\left(M_{V}<-21.5\right.$; panel a) and 65 above a fixed mass limit $(\log M>10.6$; panel b). For comparison, the parent galaxy distribution (dashed histogram) is normalized to match the AGNs in each panel. The decline in the AGN fraction toward the bluest colors (panel $a$ ) appears to be due to the inclusion of galaxies with low mass-to-light ratios that are not present in the mass-selected sample (panel $b$ ). 
remains the possibility that the lower fraction of red galaxies hosting AGN is due to some form of self-regulating feedback that effectively reduces the AGN luminosity below our flux limit. Nonetheless, these results lend no support for a simple model prescription that attributes the truncation of star formation or the color evolution of galaxies to AGN feedback and bring into question whether studies based on the optical emission line diagnostics of AGN activity miss a significant number of those residing in star-forming galaxies; see Schawinski et al. (2007) for a discussion on this topic.

\section{AGN-STAR FORMATION CONNECTION AND CO-EVOLUTION}

Having established an association between AGN activity and star formation, we are motivated to determine how closely these phenomena are related on a case-by-case basis that can potentially signify an underlying causal connection. The existence of such a relationship may be realized given the recent findings that AGN accretion power as probed by [O III] luminosity is higher for galaxies with younger stellar populations (Kauffmann et al. 2007). Ideally, we would like to investigate such a relation using a quantity more closely associated with the accretion process, namely X-ray emission, known to originate closer to the black hole and an indicator of the instantaneous star formation rate.

To do so, we plot in Figure 13(a) the SFRs of our AGN sample, as measured by [O II] strength, versus hard X-ray luminosity (2$10 \mathrm{keV}$ ) and inferred mass-accretion rate while implementing a bolometric correction as given in Marconi et al. (2004) and an accretion efficiency of 0.1 . We find that a weak correlation exists based upon a Pearson correlation coefficient of 0.17 and a linear fit that has a shallow slope with significant dispersion in its value $(0.28 \pm 0.22)$. We conclude that underlying complexities such as the efficiency of transferring gas to nuclear region over kiloparsec scales, and varying duty cycles for star formation and accretion may contribute to the large dispersion in this relation.

There have been some recent claims that an obscured phase, coupled with enhanced star formation, may represent an early stage in the subsequent evolution of AGN (e.g., Page et al. 2004; Alexander et al. 2005; Kim et al. 2006; Hopkins et al. 2008a; Polletta 2008). To test this scenario, we have marked those AGN in Figure 13(a) that have excessive X-ray absorption $\left(N_{\mathrm{H}} \gtrsim 10^{22} \mathrm{~cm}^{-2}\right)$ based on their hardness ratio $[H R=$ $(H-S) /(H+S)>-0.2]$ determined by the X-ray counts in the soft (S: $0.5-2.0 \mathrm{keV})$ and hard $(\mathrm{H}: 2-10 \mathrm{keV})$ bands assuming a power-law spectrum with a photon index of 1.9 at an effective redshift of $z \sim 0.7$. We find that the absorbed sources span the same range of SFR as the unabsorbed sources. A KS test give a probability of $65 \%$ that both absorbed and unabsorbed AGN could be drawn from the same parent population. These results are not dependent on the chosen division in hardness ratio. Therefore, we conclude that an amendment, such as the aforementioned evolutionary scenario, to the unification model (Antonucci 1993) for these moderate-luminosity AGNs is not supported by our findings. Although, there remains substantive evidence that enhanced star formation may be associated with nuclear obscuration for the more luminous QSOs (e.g., Page et al. 2004; Lacy et al. 2007; Zakamska et al. 2008) possibly due to different physical mechanisms for triggering mass accretion onto SMBHs.

It is of much interest to determine the relative growth rate of a galaxy and its central SMBH as a function of redshift in light of
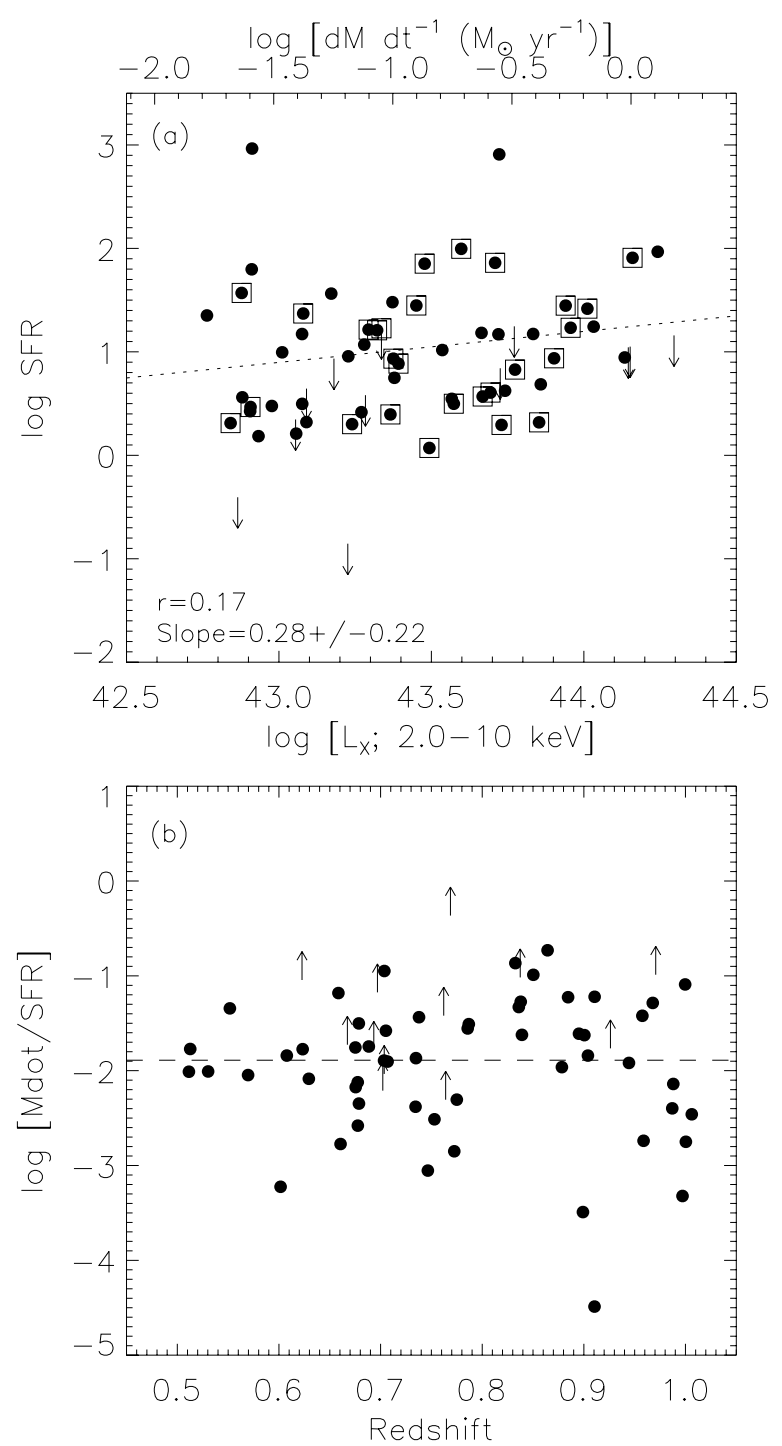

Figure 13. AGN-galaxy relations: (a) SFR vs. hard X-ray luminosity and massaccretion rate including the best-fit relation. Absorbed AGN with detected star formation are further highlighted by an open box. (b) The ratio of mass accretion to SFR vs. redshift. The horizontal dashed line marks the median ratio. Measurements are shown by a solid circle in both panels, while limits either upper $(a)$ or lower $(b)$ are given by an arrow.

the well-established local relation $M_{\mathrm{BH}} / M_{\text {bulge }} \approx 1.5 \times 10^{-3}$ (e.g., McLure \& Dunlop 2002; Haering \& Rix 2004). We plot in Figure 13(b) the ratio of mass-accretion rate onto a SMBH to the SFR as a function of redshift. The median value $\left(1.9 \times 10^{-2}\right)$ is roughly an order-of-magnitude higher than the local ratio $M_{\mathrm{BH}} / M_{\text {bulge }}$. This difference is likely due to the varying timescales between $\mathrm{SMBH}$ accretion and star formation $\Delta t_{\mathrm{BH}} / \Delta t_{\mathrm{gal}} \approx M_{\mathrm{BH}} / M_{\text {bulge }} \times S F R / d M_{\mathrm{accr}} d t^{-1} \approx 0.1$. We note that our SFR measurements most likely include a significant disk component thus a more rigorous assessment is required and beyond the scope of this work. Nonetheless, by assuming that star formation occurs over roughly a dynamical timescale $\Delta t_{\mathrm{gal}} \approx 10^{9} \mathrm{yr}$, the duty cycle for AGN activity $\left(\approx 10^{8} \mathrm{yr}\right)$ is consistent with the luminosity-dependent model predictions of Hopkins et al. (2008b) for SMBHs with $M_{\mathrm{BH}} \sim 10^{8} M_{\odot}$ and accreting above an Eddington ratio of 0.01, both within a physical regime spanned by our sample assuming these AGNs have already settled on a SMBH-bulge relation at their 
respective redshifts. Alternatively, if we assume a timescale for galaxy growth to be the inverse of their SFRs as shown in Figure 11, the AGN lifetimes can reach up to $\sim 10^{10} \mathrm{yr}$ that fully illustrates that these AGNs and their host galaxies are close to being fully matured although have SMBH growth times at least an order-of-magnitude less than those in the SDSS (Heckman et al. 2004). Furthermore, we find that there is no dependence of the ratio $\left(d M_{\mathrm{accr}} d t^{-1} / S F R\right)$ on redshift thus supporting a co-evolutionary scenario where both the average SFR and mass-accretion rates onto SMBHs are rapidly declining with equivalent rates from $z \sim 1$ to the present possibly due to diminishing fuel supplies.

These comparisons suggest that beyond the local universe the $M_{\mathrm{BH}}-M_{\text {bulge }}$ relation should display higher intrinsic dispersion given the large spread over two orders of magnitude $\left(10^{-3}\right.$ to $\left.10^{-1}\right)$ in the relation between accretion rate and SFR (Figure 13(b)). Robertson et al. (2006) point out that AGN samples at high redshift may have larger intrinsic scatter in the velocity dispersion of their hosts due to their immature dynamical state. The observational situation is so far unclear: limited AGN samples at higher redshifts (Woo et al. 2006, 2008) show preferentially higher black hole masses relative to that expected by the local $M-\sigma$ relation, while other studies of low redshift AGNs, undergoing substantial rates of accretion $\left(L_{\mathrm{Bol}} / L_{\mathrm{Edd}}>0.1\right)$, have black hole masses lower than that of inactive galaxies of equivalent host mass (Ho et al. 2008) or luminosity (Kim et al. 2008). Notwithstanding the large observational biases (Lauer et al. 2007), it is possible that the coeval growth of galaxies and their SMBHs may be intermittent with either stars or SMBHs growing somewhat faster or slower than each other, as indicated by our findings exemplified in Figure 13(b), while still resulting in a tight $\mathrm{BH}$-bulge mass relation for inactive galaxies at $z=0$ as suggested by Woo et al. (2006).

\section{SUMMARY AND CONCLUSIONS}

We have utilized the zCOSMOS $10 \mathrm{k}$ catalog of galaxies with reliable spectroscopic redshifts to investigate the properties of those that host AGN. X-ray observations with XMM-Newton enable us to identify 152 AGN, from a parent sample of 7543 zCOSMOS galaxies, that include those with significant obscuration and of low optical luminosity. The derived properties of the full zCOSMOS sample such as stellar mass, rest-frame colors, and spectral properties (i.e., emission line strength, $D_{n} 4000$ ) enable us to determine the prevalence of AGN activity as a function of galaxies with the aforementioned characteristics.

Specifically, we measure the SFR of galaxies using the $[\mathrm{O}$ II] $]$ 3727 line luminosity. We account for the contribution from the underlying AGN component most likely arising from the narrow-line region by using the [O III] $\lambda 5007$ luminosity and the typical $[\mathrm{OII}] /[\mathrm{O} \mathrm{III}]$ ratio found from previous studies of AGN and quasars (Ho 2005; Kim et al. 2006). The [O III] line luminosity is measured directly from our spectra if present. For the subsample with [O III] outside our observed spectral bandpass, we infer the [O III] strength from the hard (2-10 keV) $\mathrm{X}$-ray luminosity and the well known correlation between these two quantities.

Overall, we find that the two main requirements for a galaxy to host an actively, accreting SMBH are (1) its mass and (2) a significant amount of gas content as inferred by the observed levels of star formation and the age of their stellar populations. These findings essentially extend those of the SDSS (Kauffmann et al. 2003b) out to $z \sim 1$.
We particularly draw the following conclusions.

1. We confirm with many previous studies that the fraction of galaxies hosting AGN rises with increasing mass with most host galaxies having $M_{*}>4 \times 10^{10} M_{\odot}$.

2. The host galaxies of $A G N$ have ongoing star formation with a broad range of rates $\left(\approx 1-100 M_{\odot} \mathrm{yr}^{-1}\right)$ higher than that of the overall massive $(\log M>10.6)$ galaxy population and essentially equivalent to those forming stars (i.e., emission-line galaxies). The association of AGN activity and young stellar populations is further substantiated by an observed increase in the fraction of galaxies harboring AGN at low values of the spectral index $D_{n}(4000)$ and blue rest-frame color $U-V$.

3. The enhancement of AGN in young, star-forming galaxies with $1.0<D_{n}(4000)<1.4$ and $0.8<U-V<1.5$ lessens the evidence for AGN preferring to reside in galaxies of intermediate colors and their role in galaxy evolution. We demonstrate that previous studies based on luminosityselected samples are misleading due to the inclusion of galaxies with low mass-to-light ratios that are less likely to harbor AGN given their lower masses.

4. The SFRs of AGN hosts evolves with redshift in an equivalent manner to the overall star-forming galaxy population. This essentially brings the evidence for a co-evolution scenario between accretion onto SMBHs and the starformation history of galaxies to a closer physical scale (i.e., within the same galaxies).

5. A direct relationship between the consumption of gas into stars and that accreted onto SMBHs is weak, suggesting that additional physical complexities or varying timescales may be inherent. On average, a co-evolution scenario for the overall population is clearly evident given the constancy of the ratio $\left(\sim 10^{-2}\right)$ between mass-accretion rate onto SMBHs and SFR with redshift possibly indicative of depleting gas reservoirs from $z \sim 1$ to the present. The order-of-magnitude increase in this ratio compared to the locally measured value of $M_{\mathrm{BH}} / M_{\text {bulge }}$, is consistent with an AGN lifetime substantially shorter than that of star formation. Furthermore, the significant dispersion in this ratio may be indicative of larger scatter in the $\mathrm{BH}-$ bulge relations at higher redshift.

Our results put important constraints on physical models of AGNs and their evolution. The considerable rates of star formation $\left(\sim 10 M_{\odot} \mathrm{yr}^{-1}\right)$ in their hosts, coupled with the lack of structural signs of galaxy interactions (Grogin et al. 2005; Gabor et al. 2009) indicate that a "Seyfert mode" of accretion, driven by secular processes, is more likely than merger-driven models for this class of moderate-luminosity AGN. In particular, there is no indication of the suppression or truncation of star formation at levels expected from models implementing AGN feedback. Therefore, we find no conclusive evidence that AGNs, undergoing this mode of accretion, are a key factor in the evolution of galaxies.

We are most grateful to the referee for providing a critical review that significantly improved the paper and to Minjin Kim for allowing us to use his emission-line measurements of SDSS AGNs and fielding subsequent communications. This work is fully based on observations undertaken at the European Southern Observatory (ESO) Very Large Telescope (VLT) under the Large Program 175.A-0839 (PI: S. Lilly).

Facilities: XMM, VLT:Melipal (VIMOS) 


\section{REFERENCES}

Alexander, D. M., Smail, I., Bauer, F. E., Chapman, S. C., Blain, A. W., Brandt, W. N., \& Ivison, R. J. 2005, Nature, 434, 738

Alonso-Herrero, A., Pérez-González, P. G., Rieke, G., Alexander, D. M., Rigby, J. R., Papovich, C., Donley, J., \& Rigopoulou, D. 2008, ApJ, 677, 127

Antonucci, R. 1993, ARA\&A, 31, 473

Ballantyne, D. R., \& Papovich, C. 2007, ApJ, 660, 988

Ballantyne, D. R. 2008, ApJ, 685, 787

Balogh, M. L., Morris, S. L., Yee, H. K. C., Carlberg, R. G., \& Ellingson, E. 1999, ApJ, 527, 54

Barger, A. J., et al. 2003, AJ, 126, 632

Best, P. N., Kauffmann, G., Heckman, T. M., Brinchmann, J., Charlot, S., \& Ivezić, Ž. 2005, MNRAS, 362, 25

Böhm, A., \& Wisotzki, L. 2007, ASP Conf. Ser. 379, Cosmic Frontiers, ed. N. Metcalfe \& T. Shanks (San Francisco, CA: ASP), 185

Boyle, B. J., \& Terlevich, R. J. 1998, MNRAS, 293, L49

Brandt, W. N., \& Hasinger, G. 2005, ARA\&A, 43, 827

Brinchmann, J., Charlot, S., White, S. D. M., Tremonti, C., Kauffmann, G., Heckman, T., \& Brinkmann, J. 2004, MNRAS, 351, 1151

Bruzual, G., \& Charlot, S. 2003, MNRAS, 344, 1000

Brusa, M., et al. 2007, ApJS, 172, 353

Bundy, K., et al. 2008, ApJ, 681, 931

Calzetti, D., Armus, L., Bohlin, R. C., Kinney, A. L., Koornneef, J., \& StorchiBergmann, T. 2000, ApJ, 533, 682

Capak, P., et al. 2007, ApJS, 2007, 172, 99

Cappelluti, N., et al. 2007, ApJS, 172, 341

Cavaliere, A., \& Menci, N. 2007, ApJ, 664, 47

Cooper, M. C., et al. 2007, MNRAS, 383, 1058

Croom, S. M., et al. 2002, MNRAS, 337, 275

Croton, D. J., et al. 2006, MNRAS, 365, 11

Elvis, M., et al. 2009, ApJS, in press (arXiv:0903.2062)

Ferland, G. J., \& Osterbrock, D. E. 1986, ApJ, 300, 658

Fiore, F., et al. 2009, ApJ, 693, 447

Franceschini, A., Hasinger, G., Miyaji, T., \& Malquori, D. 1999, MNRAS, 310, L5

Gabor, J. M., et al. 2009, ApJ, 691, 705

Georgakakis, A., et al. 2008, MNRAS, 385, 2049

Gilli, R., Comastri, A., Vignali, C., \& Hasinger, G. 2007, A\&A, 463, 79

González Delgado, R. M., Heckman, T., \& Leitherer, C. 2001, ApJ, 546, 845

Granato, G. L., De Zotti, G., Silva, L., Bressan, A., \& Danese, L. 2004, ApJ, 600,580

Grogin, N. A., et al. 2005, ApJ, 627, 97

Gu, Q., et al. 2006, MNRAS, 366, 480

Haering, N., \& Rix, H.-W. 2004, ApJ, 604, 89

Hasinger, G., et al. 2007, ApJS, 172, 29

Heckman, T. M., \& Kauffmann, G. 2006, New Astron. Rev., 50, 677

Heckman, T. M., Kauffmann, G., Brinchmann, J., Charlot, S., Tremonti, C., \& White, S. D. M. 2004, ApJ, 613, 109

Heckman, T. M., Ptak, A., Hornschemeier, A., \& Kauffmann, G. 2005, ApJ, 634,161

Ho, L. 2005, ApJ, 629, 680

Ho, L. C., Darling, J., \& Green, J. E. 2008, ApJS, 177, 103

Hopkins, A. M., \& Beacom, J. F. 2006, ApJ, 651, 142

Hopkins, P. F., Bundy, K., Hernquist, L., \& Ellis, R. S. 2007, ApJ, 659, 976

Hopkins, P. F., Cox, T. J., Keres, D., \& Hernquist, L. 2008, ApJS, 175, 390

Hopkins, P. F., \& Hernquist, L. 2006, ApJS, 166, 1

Hopkins, P. F., \& Hernquist, L. 2008, arXiv:0809.3789

Jahnke, K., Kuhlbrodt, B., \& Wisotzki, L. 2004, MNRAS, 352, 399

Jahnke, K., et al. 2004, ApJ, 614, 568

Kauffmann, G., et al. 2003a, MNRAS, 341, 33

Kauffmann, G., et al. 2003b, MNRAS, 346, 1055

Kauffmann, G., et al. 2007, ApJS, 173, 357

Kewley, L. J., Geller, M. J., \& Jansen, R. A. 2004, AJ, 127, 2002

Kewley, L J., Groves, B., Kauffmann, G., \& Heckman, T. 2006, MNRAS, 372 961

Kim, M., Ho, L., \& Im, M. 2006, ApJ, 642, 702

Kim, M., Ho, L., Peng, C.. Y., Barth, A. J., Im, M., Martini, P., \& Nelson, C. H. 2008, ApJ, 687, 767

Kormendy, J., \& Kennicutt, R. C. 2004, ARA\&A, 42, 603
Lacy, M., Sajina, A., Petric, A. O., Seymour, N., Canalizo, G., Ridgway, S. E., Armus, L., \& Storrie-Lombardi, L. J. 2007, ApJ, 669, L61

Lamareille, F., et al. 2009, A\&A, 495, 53

Lauer, T. R., Tremaine, S., Richstone, D., \& Faber, S. M. 2007, ApJ., 670, 249

LaValley, M., Isobe, T., \& Feigelson, E. D. 1992, Astron. Data Anal. Softw. Syst., 1,245

Lehmer, B. D., et al. 2007, ApJ, 657, 681

Lehmer, B. D., et al. 2008, ApJ, 681, 1163

Letawe, G., Magain, P., Courbin, F., Jablonka, P., Jahnke, K., Meylan, G., \& Wisotzki, L. 2007, MNRAS, 378, 83

Lilly, S. J., Tresse, L., Hammer, F., Crampton, D., \& Le Fèvre, O. 1995, ApJ, 455,108

Lilly, S. J., et al. 2007, ApJS, 172, 70

Maier, C., et al. 2009, ApJ, 694, 1099

Mainieri, V., et al. 2007, ApJS, 172, 368

Marconi, A., Risaliti, G., Gilli, R., Hunt, L. K., Maiolino, R., \& Salvati, M. 2004, MNRAS, 351, 169

Martin, D. C., et al. 2007, ApJS, 173, 342

McLure, R. J., \& Dunlop, J. S. 2002, MNRAS, 331, 795

Merloni, A., Rudnick, G., \& Di Matteo, T. 2004, MNRAS, 354, 37

Mihos, C. J., \& Hernquist, L. 1996, ApJ, 464, 641

Moustakas, J., Kennicutt, R. C., \& Tremonti, C. A. 2006, ApJ, 642, 775

Nandra, K., et al. 2007, ApJ, 660, L11

Netzer, H., et al. 2007, ApJ, 666, 806

Noeske, K. G., et al. 2007, ApJ, 660, L43

Page, M. J., Stevens, J. A., Ivison, R. J., \& Carrera, F. J. 2004, ApJ, 611, L85

Panessa, F., Bassani, L., Cappi, M., Dadina, M., Barcons, X., Carrera, F. J., Ho, L. C., \& Iwasawa, K. 2006, A\&A, 455, 173

Pierce, C., et al. 2006, ApJ, 660, L19

Polletta, M., et al. 2008, A\&A, 492, 81

Pozzetti, L., et al. 2007, A\&A, 474, 443

Robertson, B., Hernquist, L., Cox, T. J., Di Matteo, T., Hopkins, P. F., Martini, P., \& Springel, V. 2006, ApJ, 641, 90

Salim, S., et al. 2007, ApJS, 173, 267

Sanchez, S. F., et al. 2004, ApJ, 614, 586

Sanders, D., et al. 2007, ApJS, 172, 86

Schawinski, K., et al. 2007, MNRAS, 382, 1415

Schweizer, M. Lutz, D., Sturm, E., Contursi, A., Tacconi, L. J., Lehnert, M., Dasyra, K., \& Genzel, R. 2006, ApJ, 649, 79

Scoville, N., Frayer, D. T., Schinnerer, E., \& Christopher, M. 2003, ApJ, 585, L105

Scoville, N., et al. 2007, ApJS, 172, 1

Shankar, F., Weinberg, D. H., \& Miralda Escude, J. 2009, ApJ, 690, 20

Shinozaki, K., Miyaji, T., Ishisaki, Y., Ueda, Y., \& Ogasaka, Y. 2006, AJ, 131 2843

Silverman, J. D., et al. 2005, ApJ, 618, 123

Silverman, J. D., et al. 2008a, ApJ, 675, 1025

Silverman, J. D., et al. 2008b, ApJ, 679, 118

Silverman, J. D., et al. 2009, ApJ, 695, 171

Springel, V., Di Matteo, T., \& Hernquist, L. 2005, ApJ, 620, 79

Stasińska, G., Cid Fernandes, R., Mateus, A., \& Sodré, L., Jr. 2006, MNRAS, 371,972

Sturm, E., Hasinger, G., Lehmann, I., Mainieri, V., Genzel, R., Lehnert, M. D. Lutz, D., \& Tacconi, L. J. 2006, ApJ, 642, 81

Szokoly, G. P., et al. 2004, ApJS, 155, 271

Terlevich, E., Diaz, A. I., \& Terlevich, R. 1990, MNRAS, 242, 271

Tozzi, P., et al. 2006, A\&A, 451, 457

Tresse, L., et al. 2007, A\&A, 472, 403

Trump, J., Impey, C. D., \& McCarthy, P. J. 2007, ApJS, 172, 383

Whittle, M., \& Nelson, C. 2003, ASP Conf. Ser. 297, Star Formation Through Time: A Conf. to Honour Roberto J. Terlevich, ed. E. Perez, R. M. Gonzalez Delgado, \& G. Tenorio-Tagle (San Francisco, CA: ASP), 433

Wild, V., Kauffmann, G., Heckman, T., Charlot, S., Lemson, G., Brinchmann, J., Reichard, T., \& Pasquali, A. 2007, MNRAS, 381, 543

Woo, J.-H., Treu, T., Malkan, M. A., \& Blandford, R. 2006, ApJ, 645, 900 Woo, J.-H., Treu, T., Malkan, M. A., \& Blandford, R. 2008, ApJ, 681, 925 Yan, R., et al. 2006, ApJ, 648, 281

Zakamska, N. L., Gomez, L., Strauss, M., \& Krolik, J. H. 2008, AJ, 136, 1607

Zakamska, N. L., et al. 2003, AJ, 126, 2125

Zheng, X. Z., et al. 2007, ApJ, 661, L41 